\title{
Microbial biomass and community structures in the burrows of bromophenol producing and non-producing marine worms and surrounding sediments
}

\author{
Charles C. Steward ${ }^{1}$, Stephen C. Nold ${ }^{2}$, David B. Ringelberg ${ }^{2}$, David C. White ${ }^{2}$, \\ Charles R. Lovell ${ }^{1, *}$ \\ 'Department of Biological Sciences, University of South Carolina, Columbia, South Carolina 29208, USA \\ ${ }^{2}$ Center for Environmental Biotechnology, University of Tennessee, Knoxville, Tennessee 37932, USA
}

\begin{abstract}
Microbial biomass and community structures were determined in sediments lining the burrows of 3 species of marine worms, and in nearby surface and subsurface sediments, using esterlinked phospholipid fatty acid (PLFA) analysis. The potential impact of biogenic bromophenols produced by 2 of these animals on burrow microbial communities was of particular interest. The burrow microbial communities were markedly different from those of surrounding surface and subsurface sediments. Differences in microbial biomass were attributed to burrow structures, textures of the burrow lining sediments, and organic carbon content. No significant reduction of microbial biomass or of several distinctive signature PLFA was detected in bromophenol-contaminated burrows, when compared to non-bromophenol-containing burrows. All 3 types of sediments (burrow, surface, and subsurface) examined for each worm species were distinct as determined by multivariate cluster analysis of PLFA profiles. Signature lipid biomarker PLFA for Gram-negative bacteria, Gram-positive bacteria, sulfatereducing bacteria and anaerobes, and microeucaryotes were readily identified in burrow sediments from bromophenol producing and non-producing worms.
\end{abstract}

KEY WORDS: Micrabial communities Infaunal worms Phospholipid fatty acids - Marine sediment

\section{INTRODUCTION}

Marine infauna modify sediments through burrow formation, burrow irrigation, defecation, locomotion, and excretion of soluble and insoluble exudates, resulting in physical and chemical alteration of the local sediment environment (Aller 1978, 1983, Beukema \& de Vlas 1979, Beukema 1982, Woodin et al. 1987). One important result of the construction and irrigation of burrows is formation of radial chemoclines of oxidized and reduced solutes, analogous to the vertical discontinuities seen at the sediment-water interface (Fenchel \& Reidel 1970, Aller \& Yingst 1978, Aller

\footnotetext{
- Addressee for reprint requests.
}

E-mail: lovell@biol.scarolina.edu
1980, Boudreau \& Marinelli 1994). These environmental modifications significantly affect resident microbial communities, with the most pronounced impacts occurring in sediments lining the infaunal burrows (Dobbs \& Guckert 1988b, Jensen et al. 1992) where steep gradients of potential electron donors and acceptors overlap (Aller 1983). The unique physical and chemical character of infaunal burrow linings is fairly stable compared to the more frequently disturbed sediment-water interface sediments, resulting in formation of microbial communities fundamentally different from those of nearby surface and subsurface sediments.

Microbial communities which line burrows are most accurately described as biofilms, complex accretions of microorganisms and exopolymers (Lappin-Scott \& Costerton 1989). A variety of features of the burrow 
microenvironment contribute to defining the extent and composition of the biofilms which line the burrows. Infaunal organisms frequently process the sediment in the burrow lining, altering particle size distribution (Aller 1978, 1983). Available surface area, burrow wall permeability, and fluxes of nutrients through the burrow wall are all dependent on the structure of the burrow and on the macrofaunal organism inhabiting it. The chemical environment of the burrow is also strongly affected by the macrofaunal inhabitant. Mucopolysaccharides and a wide variety of low molecular weight compounds can be produced abundantly within the burrows, presumably affecting burrow microbial communities either positively or negatively (Sheikh \& Djerassi 1975, Aller 1983, Schottler et al. 1983, King 1986, Woodin et al. 1987, Jensen et al. 1992). Which of these burrow and infaunal characteristics is most important in determining the character of the burrow lining biofilm is presently unknown.

Of the many burrow physico-chemical properties and infaunal activities which may affect the formation and character of the burrow lining biofilm, exudation of soluble metabolites is of narticular interest. A variety of infaunal polychaetes and hemichordates excrete halogenated secondary metabolites (Ashworth \& Cormier 1967, Higa \& Scheuer 1975a, b, Sheikh \& Djerassi 1975, King 1986, Woodin et al. 1987, Corgiat et al. 1993). These organisms contaminate their environs with toxic and odoriferous bromoaromatics (King 1986, Woodin et al. 1987, Steward et al. 1992, D. E. Lincoln, K. T. Fielman, R. A. Marinelli \& S. A. Woodin unpubl.), which reach their highest concentrations in the burrow lining (King 1986, Lincoln et al. unpubl.). Worm bromometabolites have been thought to serve as antifouling agents on the surfaces of the worms and within their burrows, restricting bacterial growth, oxygen consumption, degradation of the burrow wall mucopolysaccharides, and potentially preventing infection of wounds in the worms (Ashworth \& Cormier 1967 , Sheikh \& Djerassi 1975, King 1986, Goerke \& Weber 1991). However, previous studies have shown no significant impact of biogenic bromophenols on bacteria and microalgae in contaminated wormbed sediments (Jensen et al. 1992, Steward et al. 1992). The relative importance of these potential antimicrobial compounds, as well as other features of the burrow microenvironment, in defining burrow biofilm microbial communities could best be assessed by direct examination. Such investigations are complicated by the small amount of sample material which can be collected from a burrow, the large number of samples which must be analyzed to account for inherent variability among samples, and disturbance artifacts which may be introduced by sampling and sample incubation (Findlay et al. 1990).
One approach which overcomes most of these problems is extraction and analysis of cellular components, such as phospholipid fatty acids (PLFA). PLFA are found in all bacterial and eucaryotic membranes and provide a basis for the determination of viable microbial biomass and a definition of microbial community structures in marine sediments in situ (Perry et al. 1979, White et al. 1979, Gillan et al. 1983, Baird \& White 1985, Baird et al. 1985, Guckert et al. 1985, Currie \& Johns 1988, Findlay et al. 1990, Rajendran et al. 1992). Since phospholipids are not used as reserve polymers and are rapidly turned over in marine sediments (White 1983, 1988) they provide a sensitive measure of the viable microbial biomass. Analysis of membrane-bound (ester-linked) PLFA after their conversion to fatty acid methyl esters assures that only PLFA derived from viable organisms are included in the analysis. Samples for PLFA analysis can be collected and immediately frozen in the field, minimizing disturbance artifacts (Findlay et al. 1990). PLFA can also be extracted from small samples, and large sample sets can be easily and reproducibly analyzed providing a sensitive and quảntititalive means for studying the in situ microbial biomass and community structure. Multivariate statistical analysis of PLFA profiles allows quantitative comparisons between different sample types and sample groups. While the method does not allow complete identification or quantification of all microbial groups in complex communities (see Haack et al. 1994), it has proven very useful in the determination of microbial biomass and identification of some taxonomic and functional groups in environmental samples. PLFA-based estimates of biomass have been shown to correspond to estimates based on other measures, including direct microscopic counts (Balkwill et al. 1988, White 1988), and a number of different microbial groups contain useful indicator PLFA (Federle et al. 1986, Vestal \& White 1989, Tunlid \& White 1992). We have examined microbial biomass and community structure in sediments lining the burrows of 3 marine worms, comparing burrow microbial communities to those in nearby surface and subsurface sediments. Of particular interest were the impacts of biogenic bromophenols and other characteristics of the burrow lining microenvironment on microbial biomass and community structure.

\section{MATERIALS AND METHODS}

Materials. Solvents from Burdick \& Jackson (Mukegon, $\mathrm{MI}, \mathrm{USA})$ were residue analysis grade $\left(\mathrm{GC}^{2}\right)$. Standards and derivatizing reagents were purchased from Supelco Inc. (Bellefonte, PA, USA), Nu Chek Prep (Elysian, MN, USA) and Pierce Chemical Co. (Rock- 
ford, IL, USA). Bromophenols were obtained from Aldrich Chemical Co. (Milwaukee, WI, USA) and Sigma (St. Louis, MO, USA). The purity of each compound was evaluated using gas chromatography/mass spectroscopy (GC/MS) and pure lots used without further purification.

Study site. The North Inlet estuary $\left(33^{\circ} 20^{\prime} \mathrm{N}, 79^{\circ} 10^{\prime} \mathrm{W}\right)$ is a relatively small (3200 ha), bar-built Spartina marsh system located near Georgetown, South Carolina, USA. Dame et al. (1986) and Pinckney \& Zingmark (1993) have provided detailed descriptions of this system. The capitellid polychaete Notomastus lobatus, maldanid polychaete Branchyoasychus americana, and hemichordate Balanoglossus aurantiacus are abundant in North Inlet intertidal sandflats, and their burrows can be recognized on site by their characteristic fecal mounds, burrow openings, or tube structures (Ruppert \& Fox 1988). All are deposit feeders; $N$ lobatus and $B$. americana are head down subsurface deposit feeders. The $B$. americana tube is straight and vertical with the head shaft opening 25 to $30 \mathrm{~cm}$ below the sediment surface. This maldanid prefers small particles in tube construction and is often associated with clumps of more cohesive mud within sand-and mudflats (Mangum 1964a). It appears to irrigate weakly, if at all, and does not create a well-aerated microenvironment within the tube (Mangum 1964b). The upper 6 to $10 \mathrm{~cm}$ of $N$. lobatus burrows are straight and vertical. Below this point, the burrow becomes helical (Powell 1977), a unique structure at this sampling site. No observations of $N$. lobatus irrigation behavior have been reported, but the light brown oxidized sediment layer lining the burrow is quite thin, likely indicating burrow water stagnation. B. aurantiacus builds a U-shaped burrow, excavating a shallow subsurface feeding chamber at the head end (Rupert \& Fox 1988). The surface sediments overlying this chamber slump, forming a small, shallow basin into which flocculent detrital materials collect and are ingested by the worm. Burrow irrigation data are not available, but the sediments lining the burrow are light colored and appear to be highly oxidized.

Sample collection. Sediment samples for PLFA analysis were collected as matched sets, with each including 1 worm burrow sediment sample, 1 surface sediment sample, and 1 subsurface sediment sample. Subsurface samples were taken approximately 10 to $20 \mathrm{~cm}$ deep, near but not adjacent to worm burrows. Surface sediments were collected from approximately the top $1 \mathrm{~cm}$ of surface sediment near but not adjacent to the worm burrows. Sediments (approximately 1 to $2 \mathrm{~mm}$ thickness) were scraped from the linings of the burrows for Notomastus lobatus and Balanoglossus aurantiacus using sterile spatulas, and pieces of Branchyoasychus americana tubes were recovered intact.
Subsurface and surface sediments were also collected using sterile spatulas. Large macrofauna were excluded from all samples. Ten sets were collected from burrows and surrounding sediments for each worm species. In all cases, positive identification of each worm was made after exhuming an identifiable portion or intact individual. The samples were placed in sterile Whirl-pak (Nasco, Ft. Atkinson, WI, USA) bags and immediately frozen on dry ice for transport to Columbia, South Carolina. Samples were stored at $-80^{\circ} \mathrm{C}$ pending transport to Knoxville, Tennessee, USA, for extraction and analysis. Integrated bulk wormbed sediments were collected using cut-off plastic syringes (2.6 cm diameter, $11 \mathrm{~cm}$ long), and burrow sediment samples were collected as described above for sediment analyses. Samples of $B$. americana tissue were collected and stored in methanol (Burdick \& Jackson, Nanograde) for extraction of halogenated aromatic compounds for analysis by GC/MS.

Sediment extraction and lipid analysis. The 1 to $20 \mathrm{~g}$ sediment samples were extracted and PLFA recovered and derivatized for identification and quantification as described by Guckert et al. (1991). Samples (1.0 $\mu \mathrm{l})$ were injected onto the column of an IBM gas chromatograph (GC) equipped with a $60 \mathrm{~m} \mathrm{Rt}_{\mathrm{x}}-1$ (nonpolar methyl silicone) column with a $30 \mathrm{~s}$ splitless injection time and an injection temperature of $290^{\circ} \mathrm{C}$. Phospholipid fatty acid methyl esters (PLFAME) were resolved using a temperature program of $100^{\circ} \mathrm{C}$ to $150^{\circ} \mathrm{C}$ at $10^{\circ} \mathrm{C} \mathrm{min}^{-1}, 150^{\circ} \mathrm{C}$ for $1 \mathrm{~min}, 150^{\circ} \mathrm{C}$ to $282^{\circ} \mathrm{C}$ at $3^{\circ} \mathrm{C} \mathrm{min}^{-1}$, and $282^{\circ} \mathrm{C}$ for $5 \mathrm{~min}$. Hydrogen (linear velocity $35 \mathrm{~cm} \mathrm{~s}^{-1}$ ) was the carrier gas. Detection was

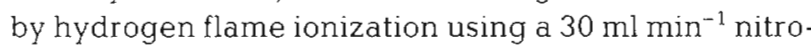
gen makeup gas at a temperature of $290^{\circ} \mathrm{C}$. An equal detector response was assumed for all components. Peak areas were quantified using the PE Nelson 3000 series Chromatography Data System (Revision 5.0). Determination of fatty acid structures was performed using GC/MS as described by Ringelberg et al. (1986). Bacterial fatty acid double bond position and geometry were confirmed using GC/MS analysis of the dimethyl disulfide adducts of the monounsaturated PLFAME as described in Nichols et al. (1986). Additional verification was done, as needed, using equivalent chain length analysis (Christie 1989).

Fatty acid nomenclature. Fatty acids are designated as $A: B \omega C$, where $A$ is the total number of carbon atoms, $B$ is the number of double bonds, and $C$ is the position of the double bond from the aliphatic end of the molecule. Geometry of this bond is indicated ' $c$ ' for cis and ' $t$ ' for trans. The prefixes ' $\mathrm{i}$ ' and ' $\mathrm{a}$ ' refer to iso and anteiso methyl branching respectively (Kates 1986). Mid-chain methyl branches are designated by 'me' preceded by the position of the methyl group from the acid end of the molecule. Cyclopropyl fatty acids are designated as ' $c y$ '. 
Worm halometabolite analysis. Methanol extracts from Branchyoasychus americana, which had not been previously screened for production of halometabolites, were filtered through $0.2 \mathrm{\mu m}$ filters to remove particulates. Extracts were examined for halometabolites using GC/MS as described previously (Woodin et al. 1993)

Sediment grain size and carbon analysis. Burrow lining and bulk wormbed sediments were dried to constant weight at $70^{\circ} \mathrm{C}$ and sized through a Wentworth series of sieves, using a mechanical shaker (Tyler. Industrial Products, Mentor, OH, USA) to facilitate size fractionation. Percentage silt and clay was measured as the portion of sediment with a grain size $\leq 63 \mu \mathrm{m}$. Organic carbon content of wormbed and burrow sediment was determined as ash free dry weight following combustion at $500^{\circ} \mathrm{C}$ in a muffle furnace

Statistical analysis. PLFA profiles were checked for normality of distribution (variances were homogeneous), biomass data were log transformed, and PLFA mole percentage data were $\arcsin \left(V_{x}\right)$ transformed prior to statistical analysis. Analysis of PLFA data was performed using multivariate rluster analysis, principal components analysis, and analysis of variance (ANOVA), TukeyKramer test for Honestly Significant Difference (Tukey test), and discriminant analysis programs in commercially available software packages: Ein-sight (Infometrix, Seattle, WA, USA), Statgraphics (Statistical Graphics Corporation, Rockville, MD, USA), and SAS (SAS Institute, Cary, NC, USA). Residual plots indicated homogeneous variances. A significance level of $p \leq 0.01$ was used for all analyses following a power test which showed very large power of analyses with these data.

\section{RESULTS AND DISCUSSION}

\section{Characteristics of sediments and worm burrows}

Sediments surrounding and within burrows of the 3 species of marine worms differed substantially (Table 1). Notomastus lobatus lives in fine-grained muddy sands. Its burrow wall sediments were tightly compacted, cohesive, polished, heavily coated with biogenic films of mucus, and organically enriched. Looking from the burrow outward into the surrounding anoxic sediment, the transition from a thin layer of oxidized (light brown) burrow lining sediment to black anoxic sediment was very apparent. Balanoglossus aurantiacus lives in coarser muddy sands with lower silt and clay content than N. Iobatus or Branchyoasychus americana. The sediments lining these burrows were not very cohesive, had only a light coating of mucus, and were much lower in organic carbon than the other two types of worm burrows. B. americana builds a highly structured tube in fine sand and muds similar to those inhabited by $N$. lobatus. A portion of the tube structure usually extends above the sedimentwater interface. Sediments lining these tubes are firmer than the surrounding sediment, very cohesive, and the tube can often be removed from the sediment intact. $B$. americana tube sediments are enriched in organic carbon. The inner lining of the tube is highly polished, with mucus present. The surface sediments at all locations were fine in texture and highly oxidized, as indicated by their light brown color. Previous studies have shown sediments in these and similar locations to contain high levels of diatom biomass (Steward et al. 1992, Pinckney \& Zingmark 1993). All subsurface sediments were black, indicating anoxia and chemical reduction. Organic content of all integrated surface and subsurface sediments was lower than that of burrow sediments.

\section{Bromometabolite analysis}

Bromometabolites produced by these species have been identified by gas chromatography/mass spectroscopy, either previously or for this study. Notomastus lobatus produces 4-bromophenol, 2,4-dibromophenol, and 2,4,6-tribromophenol (Chen et al. 1991, Steward et al. 1992). Balanoglossus aurantiacus produces 2,6dibromophenol (Steward et al. 1995). This compound

Table 1. Median grain size, \% silt and clay, and organic carbon content for wormbed sediments collected around each of 3 worm species, and median grain size, \% silt and clay, and organic carbon content for worm burrow lining sediments. Values are given as mean $\pm \mathrm{SD}(\mathrm{n}=10)$ for each set of samples

\begin{tabular}{|lccc|}
\hline Location & Median grain size $(\mu \mathrm{m})$ & Silt and clay $(\%)$ & Organic C $(\%)$ \\
\hline Balanoglossus aurantiacus wormbed & $191 \pm 2$ & $0.9 \pm 0.2$ & $0.95 \pm 0.08$ \\
Notomastus lobatus wormbed & $167 \pm 1$ & $3.1 \pm 1.4$ & $2.16 \pm 0.22$ \\
Branchyoasychus americana wormbed & $163 \pm 1$ & $3.6 \pm 1.5$ & $2.04 \pm 0.47$ \\
B. aurantiacus burrow & $178 \pm 3$ & $0.7 \pm 0.2$ & $1.11 \pm 0.12$ \\
N. lobatus burrow & $162 \pm 5$ & $7.1 \pm 2.3$ & $3.90 \pm 0.56$ \\
B. americana burrow & $136 \pm 10$ & $7.9 \pm 1.2$ & $3.27 \pm 0.31$ \\
\hline
\end{tabular}


has also been identified in the closely related species Balanoglossus biminiensis (Ashworth \& Cormier 1967). $B$. aurantiacus is also sympatric with Saccoglossus kowalewskyi at this site, but is more abundant and more easily identified in the field. B. aurantiacus produces the same compound (2,6-dibromophenol) as the S. kowalewskyi used in previous studies (King 1986), while the $S$. kowalewskyi at this site produces bromopyrroles (Woodin et al. 1987, Fielman \& Target 1995). Although a different genus and species than $S$. kowalewskyi, $B$. aurantiacus has the advantages of producing the same compound, producing similar burrow structure, and living in sediments similar to those of worms used in previous studies (King 1986, 1988). No volatile halogenated aromatic compounds were detected in Branchyoasychus americana (this study); King (1986) also found no volatile halogenated compounds in a closely related species, Clymenella torquata.

\section{PLFA biomass}

Burrow lining and surface and subsurface wormbed sediment microbial communities were examined using PLFA analysis. The mean quantities of the most abundant PLFA in the profiles for each sample set and sediment type are shown in Tables 2 to 4 . Quantities are given as the mole percentage of the total amount of PLFA recovered as phospholipid fatty acid methyl esters. The transesterification process ensures recovery of only the membrane-bound PLFA, which have ester bonds linking the fatty acids to the glycerol head group. As a result, the analysis provides information primarily on viable microorganisms due to the rapid degradation of free bacterial phospholipids in marine sediments (White et al. 1979). A total of 60 fatty acids were identified in each profile, but many of these were present in very low quantities representing mole percentages less than $0.1 \%$ of the profile total. The PLFA listed in Tables 2 to 4 accounted for 93 to $97 \mathrm{~mol} \%$ of the total PLFA present in each sample.

Mean PLFA biomass was generally highest in the surface sediments, intermediate in the burrow sediments, and lowest in the subsurface sediments (Tables 2 to 4, Fig. 1). Sample groups (burrow, surface, and subsurface) and worm species associated with the samples both had significant effects (2-way ANOVA: sediment type: $\mathrm{MSE}=10.50, \mathrm{df}=2, F=139.06, \mathrm{p} \leq$ 0.0001 ; worm species: $\mathrm{MSE}=1.29, \mathrm{df}=2, F=17.13, \mathrm{p} \leq$ 0.0001 ). Further analysis of PLFA biomass estimates using a Tukey test (Fig. 1) showed some exceptions. The Notomastus lobatus burrow and surface samples were homogeneous with respect to biomass, as were the Balanoglossus aurantiacus burrow and subsurface samples. While significantly lower than the surface biomass in 2 out of 3 cases, PLFA biomass within burrows of all 3 worm species was substantial, more than 3 times the levels found in subsurface sediments. Total PLFA biomass was not significantly different (Tukey test) between the Branchyoasychus americana (no bromometabolites) and $N$. lobatus (bromophenols) burrow samples, nor between the $N$. lobatus and $B$. aurantiacus (dibromophenol) burrow samples. However, burrow microbial. biomass significantly differed between $B$. americana and B. aurantiacus, the organisms inhabiting the least similar sediments and forming the most dissimilar burrow structures (Table 1, Fig. 1).

Infaunal burrows and ventilated sediments are well known to be sites of increased microbial biomass and activity (Aller 1978, 1983, Aller \& Yingst 1978, Alongi 1985, Krantzberg 1985), most likely due to burrow properties and macrofaunal activities. Dobbs \& Guckert (1988a) used PLFA analysis to show. that the burrows of Callianassa trilobata, an infaunal shrimp, contained higher microbial biomass than surrounding sediments. The burrows used in this study, including the bromophenol-contaminated burrows, are no exception. Microbial biomass in the Notomastus lobatus burrow lining, which is exposed to $>800 \mathrm{ng} \mathrm{g}^{-1}$ dry weight sediment of bromophenols (Lincoln et al. unpubl.), was as high as that observed in the surface sediments (Tables $2 \& 3$, Fig. 1). Similarly, Jensen et al. (1992) found burrow bacterial numbers twice as high as those in subsurface control sediments. These results suggest that elevated microbial biomass in infaunal burrows is more directly attributable to the physicochemical properties of the burrow and irrigation activities of the infaunal host organism (Boudreau \& Marinelli 1994) than to the presence or absence of halo-aromatic secondary metabolites.

\section{Microbial community composition}

More detailed examination of the PLFA profiles revealed some striking differences between microbial communities in the 3 sample types (Figs. 2 \& 3). Surface sediment microbial. communities typically contained high levels of polyunsaturated phospholipid fatty acids (PUFA), including 20:4 16 and 20:5 $\omega 3$, while subsurface sediment communities had much lower levels of these PLFA. Subsurface sediments contained elevated levels of branched saturated fatty acids relative to the other sediment types (Figs. $2 \& 3$ ). PLFA structural groups recovered from burrow microbial communities were most often present at levels intermediate between those for surface and subsurface sediments.

Differences in microbial community structures were also observed among the different burrows examined 
Table 2. Phospholipid fatty acid (PLFA) values presented as a mol\% of total PLFA for burrow sediments from 3 marine worms PLFA shown are the most abundant $(>0.1 \mathrm{~mol} \%)$ fatty acids in the profiles. Sum SAFA: sum of all saturated PLFA; sum BRSAFA: sum of all terminally branched saturated PLFA; sum MUFA and Cy: sum of all monounsaturated and cyclopropyl PLFA; sum PUFA: sum of all polyunsaturated PLFA; sum meBRSAFA: sum of all methyl branched saturated PLFA; Cy/MUFA: ratio of cyclopropyl PLFA to the MUFA precursor; Trans/cis: ratio of trans to cis isomers of a given PLFA. Values presented are mean and standard deviation $(n=10)$ for each set of samples

\begin{tabular}{|c|c|c|c|c|c|c|}
\hline \multirow[t]{2}{*}{ Sample type } & \multicolumn{2}{|c|}{ Balanoglossus aurantracus } & \multicolumn{2}{|c|}{ Notomastus lobatus } & \multicolumn{2}{|c|}{ Branchyoasychus americana } \\
\hline & Mean & $\mathrm{SD}$ & Mean & $\mathrm{SD}$ & Mean & $\mathrm{SD}$ \\
\hline \multicolumn{7}{|l|}{ PLFA } \\
\hline $14: 0$ & 0.95 & 0.89 & 1.22 & 0.99 & 2.58 & 0.84 \\
\hline $15: 0$ & 1.62 & 0.66 & 0.97 & 0.44 & 1.34 & 0.45 \\
\hline $16: 0$ & 13.42 & 1.59 & 11.41 & 1.89 & 13.29 & 1.12 \\
\hline $17: 0$ & 1.79 & 0.21 & 1.33 & 0.28 & 0.82 & 0.23 \\
\hline $18: 0$ & 3.30 & 0.63 & 3.46 & 1.51 & 1.78 & 0.47 \\
\hline $20: 0$ & 0.48 & 0.07 & 0.52 & 0.22 & 0.26 & 0.07 \\
\hline $22: 0$ & 0.25 & 0.06 & 0.20 & 0.08 & 0.12 & 0.03 \\
\hline $24: 0$ & 0.31 & 0.08 & 0.18 & 0.10 & 0.15 & 0.07 \\
\hline Sum SAFA & \multicolumn{2}{|c|}{22.12} & \multicolumn{2}{|c|}{19.30} & \multicolumn{2}{|c|}{20.35} \\
\hline i14:0 & 0.19 & 0.34 & 0.41 & 0.35 & 0.66 & 0.28 \\
\hline i15:0 & 2.18 & 1.08 & 2.57 & 1.42 & 3.98 & 1.01 \\
\hline $\mathrm{a} 15: 0$ & 3.35 & 1.66 & 3.00 & 2.01 & 4.89 & 1.25 \\
\hline i16:0 & 1.10 & 0.25 & 1.14 & 0.51 & 1.34 & 0.29 \\
\hline i17:0 & 0.76 & 0.04 & 0.74 & 0.22 & 0.71 & 0.11 \\
\hline $17: 1 \omega 8 \mathrm{c} / \mathrm{a} 17: 0$ & 3.53 & 0.24 & 2.25 & 0.57 & 2.12 & 0.43 \\
\hline Sum BRSAFA & \multicolumn{2}{|c|}{11.45} & \multicolumn{2}{|c|}{8.02} & \multicolumn{2}{|c|}{14.02} \\
\hline $\mathrm{a} 16: 0 / 16: 1 \omega 9 \mathrm{C}$ & 0.59 & 0.12 & 0.89 & 0.33 & 1.03 & 0.20 \\
\hline $16: 1 \omega 7 \mathrm{c}$ & 10.92 & 2.15 & 13.94 & 5.05 & 16.77 & 1.97 \\
\hline $16: 1 \omega 7 \mathrm{t}$ & 0.37 & 0.08 & 0.42 & 0.15 & 1.06 & 0.18 \\
\hline $16: 1 \omega 5 c$ & 1.33 & 0.25 & 1.57 & 0.52 & 2.13 & 0.67 \\
\hline $16: 1 \omega 13 t$ & 0.37 & 0.09 & 0.14 & 0.07 & 0.22 & 0.12 \\
\hline $17: 1 \omega 6 \mathrm{c}$ & 1.03 & 0.21 & 0.72 & 0.24 & 0.67 & 0.14 \\
\hline $\operatorname{cy} 17: 0$ & 0.51 & 0.15 & 0.45 & 0.24 & 0.75 & 0.21 \\
\hline $18: 1 \omega 9 \mathrm{c}$ & 3.25 & 0.47 & 2.85 & 0.60 & 1.98 & 0.43 \\
\hline $18: 1 \omega 7 \mathrm{c}$ & 12.16 & 1.84 & 13.67 & 3.39 & 20.20 & 7.04 \\
\hline cy 19:0 $(\omega 7,8)$ & 0.44 & 0.08 & 0.92 & 0.67 & 4.02 & 2.83 \\
\hline Sum MUFA and Cy & \multicolumn{2}{|c|}{33.26} & \multicolumn{2}{|c|}{38.30} & \multicolumn{2}{|c|}{50.41} \\
\hline $16: 2 \omega 6 /$ br $15: 0$ & 0.49 & 0.14 & 0.31 & 0.14 & 0.33 & 0.12 \\
\hline poly 17 & 0.38 & 0.13 & 0.12 & 0.06 & 0.18 & 0.08 \\
\hline $18: 3 \omega 6$ & 0.50 & 0.08 & 0.32 & 0.19 & 0.51 & 0.13 \\
\hline $18: 4 \omega 3 / 12$ me $17: 0$ & 0.68 & 0.26 & 0.46 & 0.24 & 0.59 & 0.12 \\
\hline $18: 2 \omega 6$ & 0.59 & 0.21 & 0.88 & 0.59 & 0.32 & 0.27 \\
\hline $18: 3 \omega 3 /$ br $17: 1$ & 2.00 & 0.31 & 1.19 & 0.59 & 1.33 & 0.25 \\
\hline $20: 4 \omega 6$ & 6.44 & 1.87 & 4.26 & 1.35 & 1.77 & 1.16 \\
\hline $20: 5 \omega 3$ & 7.60 & 2.24 & 6.53 & 3.46 & 1.97 & 1.86 \\
\hline $20: 306$ & 0.41 & 0.13 & 0.26 & 0.09 & 0.14 & 0.08 \\
\hline $20: 4 \omega 3$ & 0.68 & 0.46 & 0.33 & 0.13 & 0.06 & 0.03 \\
\hline $20: 3 \omega 3$ & 0.85 & 0.34 & 2.59 & 1.78 & 0.55 & 0.70 \\
\hline $22: 5 \omega 6$ & 1.17 & 0.27 & 0.93 & 0.30 & 0.21 & 0.30 \\
\hline $22: 6 \omega 3$ & 2.41 & 0.75 & 2.75 & 1.48 & 0.72 & 0.85 \\
\hline $22: 4 \omega 6$ & 0.55 & 0.16 & 1.38 & 0.80 & 0.21 & 0.30 \\
\hline $22: 5 \omega 3$ & 0.68 & 0.18 & 1.41 & 1.65 & 0.24 & 0.43 \\
\hline poly 22 & 2.56 & 2.07 & 1.33 & 2.15 & 0.03 & 0.03 \\
\hline Sum PUFA & \multicolumn{2}{|c|}{29.11} & \multicolumn{2}{|c|}{26.28} & \multicolumn{2}{|c|}{9.58} \\
\hline $10 \mathrm{me} 16: 0$ & 2.17 & 0.19 & 3.25 & 1.31 & 3.35 & 0.69 \\
\hline 10.11 me $18: 0$ & 0.47 & 0.03 & 0.66 & 0.31 & 0.54 & 0.13 \\
\hline Sum meBRSAFA & & & & & & \\
\hline Sum of listed PLFA & & & & & & \\
\hline Total pmol g $g^{-1}$ dry wt & 11584 & 5186 & 36955 & 21731 & 55899 & 29540 \\
\hline Cy/MUFA & & & & & & \\
\hline cy $17: 0 / 16: 1 \omega 7 c$ & 0.05 & 0.01 & 0.03 & 0.02 & 0.04 & 0.01 \\
\hline cy $19: 0 / 18: 1 \omega 7 \mathrm{c}$ & 0.04 & 0.009 & 0.06 & 0.04 & 0.17 & 0.08 \\
\hline Trans/cis & & & & & & \\
\hline $16: 1 \omega 7 t / 16: 1 \omega 7 c$ & 0.03 & 0.005 & 0.03 & 0.01 & 0.06 & 0.02 \\
\hline $18: 1 \omega 7 \mathrm{t} / 18: 1 \omega 7 \mathrm{c}$ & 0.03 & 0.004 & 0.03 & 0.02 & 0.03 & 0.006 \\
\hline
\end{tabular}


Table 3. PLFA values presented as a mol\% of total PLFA for surface sediments near burrows of 3 marine worms. See Table 2 legend for details

\begin{tabular}{|c|c|c|c|c|c|c|}
\hline \multirow[t]{2}{*}{ Sample type } & \multicolumn{2}{|c|}{ Balanoglossus aurantiacus } & \multicolumn{2}{|c|}{ Notomastus lobatus } & \multicolumn{2}{|c|}{ Branchyoasychus americana } \\
\hline & Mean & $\mathrm{SD}$ & Mean & $\mathrm{SD}$ & Mean & $\mathrm{SD}$ \\
\hline \multicolumn{7}{|l|}{ PLFA } \\
\hline $14: 0$ & 4.30 & 1.58 & 2.07 & 0.96 & 7.33 & 1.14 \\
\hline $15: 0$ & 3.66 & 0.58 & 1.63 & 0.39 & 3.13 & 0.22 \\
\hline $16: 0$ & 17.45 & 0.45 & 17.30 & 1.95 & 17.08 & 0.86 \\
\hline $17: 0$ & 1.38 & 0.15 & 1.13 & 0.20 & 1.06 & 0.12 \\
\hline $18: 0$ & 2.32 & 0.32 & 2.80 & 1.32 & 1.90 & 0.19 \\
\hline $20: 0$ & 0.28 & 0.04 & 0.33 & 0.18 & 0.24 & 0.02 \\
\hline $22: 0$ & 0.22 & 0.08 & 0.21 & 0.12 & 0.17 & 0.02 \\
\hline $24: 0$ & 0.44 & 0.06 & 0.25 & 0.07 & 0.32 & 0.07 \\
\hline Sum SAFA & \multicolumn{2}{|c|}{29.82} & \multicolumn{2}{|c|}{25.73} & \multicolumn{2}{|c|}{31.23} \\
\hline i14:0 & 0.46 & 0.26 & 0.25 & 0.11 & 1.06 & 0.07 \\
\hline$i 15: 0$ & 1.95 & 0.37 & 1.15 & 0.35 & 2.54 & 0.16 \\
\hline a 15:0 & 2.40 & 0.47 & 1.37 & 0.41 & 3.05 & 0.20 \\
\hline$i 16: 0$ & 0.74 & 0.06 & 0.67 & 0.15 & 0.80 & 0.04 \\
\hline $\mathrm{i} 17: 0$ & 0.46 & 0.04 & 0.45 & 0.09 & 0.44 & 0.02 \\
\hline $17: 1 \omega 8 \mathrm{c} / \mathrm{a} 17: 0$ & 3.06 & 0.24 & 1.93 & 0.36 & 2.18 & 0.14 \\
\hline Sum BRSAFA & \multicolumn{2}{|c|}{9.09} & \multicolumn{2}{|c|}{5.80} & \multicolumn{2}{|c|}{10.07} \\
\hline a $16: 0 / 16: 1 \omega 9 c$ & 0.74 & 0.07 & 0.75 & 0.16 & 0.95 & 0.05 \\
\hline $16: 1 \omega 7 \mathrm{C}$ & 15.07 & 0.93 & 14.43 & 2.50 & 16.20 & 1.20 \\
\hline $16: 1 \omega 7 \mathrm{t}$ & 0.23 & 0.07 & 0.26 & 0.05 & 0.35 & 0.03 \\
\hline $16: 1 \omega 5 c$ & 1.23 & 0.12 & 1.04 & 0.16 & 1.27 & 0.06 \\
\hline $16: 1013 t$ & 1.17 & 0.12 & 0.96 & 0.20 & 1.16 & 0.13 \\
\hline $17: 106 \mathrm{c}$ & 0.76 & 0.12 & 0.60 & 0.11 & 0.71 & 0.10 \\
\hline $\mathrm{Cy} 17: 0$ & 0.03 & 0.07 & 0.23 & 0.07 & 0.21 & 0.03 \\
\hline $18: 1 \omega 9 c$ & 2.52 & 0.24 & 3.61 & 0.39 & 2.60 & 0.23 \\
\hline $18: 107 \mathrm{C}$ & 7.79 & 0.91 & 9.35 & 1.19 & 7.55 & 0.56 \\
\hline 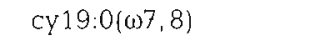 & 0.15 & 0.06 & 0.38 & 0.13 & 0.18 & 0.03 \\
\hline Sum MUFA and $C y$ & \multicolumn{2}{|c|}{31.36} & \multicolumn{2}{|c|}{33.77} & \multicolumn{2}{|c|}{32.69} \\
\hline $16: 2 \omega 6 /$ br $15: 0$ & 1.04 & 0.18 & 0.71 & 0.21 & 0.99 & 0.28 \\
\hline poly 17 & 1.07 & 0.23 & 0.55 & 0.15 & 0.58 & 0.08 \\
\hline $18: 306$ & 0.52 & 0.07 & 2.21 & 0.47 & 0.37 & 0.05 \\
\hline $18: 403 / 12$ me $17: 0$ & 1.39 & 0.15 & nd & nd & 1.28 & 0.11 \\
\hline $18: 2 \omega 6$ & 0.83 & 0.11 & 0.98 & 0.13 & 0.82 & 0.10 \\
\hline $18: 3 \omega 3 / \mathrm{br} 17: 1$ & 0.76 & 0.08 & 1.12 & 0.28 & 0.81 & 0.06 \\
\hline $20: 4 \omega 6$ & 3.31 & 0.49 & 3.51 & 0.47 & 2.67 & 0.44 \\
\hline $20: 5 \omega 3$ & 11.52 & 0.87 & 13.87 & 2.48 & 10.00 & 1.04 \\
\hline $20: 3 \omega 6$ & 0.20 & 0.08 & 0.30 & 0.04 & 0.20 & 0.04 \\
\hline $20: 4 \omega 3$ & 0.32 & 0.05 & 0.41 & 0.07 & 0.30 & 0.05 \\
\hline $20: 303$ & 0.18 & 0.09 & 0.97 & 0.65 & 0.24 & 0.07 \\
\hline $22.5 \omega 6$ & 1.02 & 0.18 & 0.78 & 0.10 & 0.79 & 0.09 \\
\hline $22: 6003$ & 4.02 & 0.45 & 5.01 & 1.37 & 3.81 & 0.55 \\
\hline $22: 4 \omega 6$ & 0.20 & 0.07 & 0.27 & 0.09 & 0.16 & 0.04 \\
\hline $22: 503$ & 0.58 & 0.12 & 0.66 & 0.25 & 0.46 & 0.12 \\
\hline poly 22 & 0.01 & 0.04 & 0.05 & 0.05 & 0.03 & 0.05 \\
\hline Sum PUFA & \multicolumn{2}{|c|}{27.81} & \multicolumn{2}{|c|}{32.29} & \multicolumn{2}{|c|}{24.13} \\
\hline 10me16:0 & 0.78 & 0.11 & 1.21 & 0.25 & 0.82 & 0.12 \\
\hline 10.11me18:0 & 0.02 & 0.03 & 0.36 & 0.05 & 0.08 & 0.03 \\
\hline Sum meBRSAFA & & & & & & \\
\hline Sum of PLFA listed & & & & & & \\
\hline Total pmol $g^{-1}$ dry $w t$ & 57996 & 14611 & 48959 & 18700 & 117459 & 24624 \\
\hline $\mathrm{Cy} / \mathrm{MUFA}$ & & & & & & \\
\hline cy $17: 0 / 16: 1 \omega 7 c$ & 0.00 & 0.004 & 0.02 & 0.007 & 0.01 & 0.004 \\
\hline 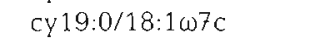 & 0.02 & 0.006 & 0.04 & 0.008 & 0.03 & 0.005 \\
\hline Trans/cis & & & & & & \\
\hline $16: 1 \omega 7 \mathrm{t} / 16: 1 \omega 7 \mathrm{C}$ & 0.02 & 0.007 & 0.02 & 0.006 & 0.02 & 0.004 \\
\hline $18: 1 \omega 7 \mathrm{t} / 18: 1 \omega 7 \mathrm{c}$ & 0.02 & 0.008 & 0.02 & 0.003 & 0.02 & 0.005 \\
\hline
\end{tabular}


Table 4. PLFA values presented as a mol\% of total PLFA for subsurface sediments near burrows of 3 marine worms. See Table 2 legend for details

\begin{tabular}{|c|c|c|c|c|c|c|}
\hline \multirow[t]{2}{*}{ Sample type } & \multicolumn{2}{|c|}{ Balanoglossus aurantacus } & \multicolumn{2}{|c|}{ Notomastus lobatus } & \multicolumn{2}{|c|}{ Branchyoasychus americana } \\
\hline & Mean & $\mathrm{SD}$ & Mean & $\mathrm{SD}$ & Mean & $\mathrm{SD}$ \\
\hline \multicolumn{7}{|l|}{ PLFA } \\
\hline $14: 0$ & 1.61 & 1.78 & 1.48 & 0.96 & 2.36 & 0.69 \\
\hline $15: 0$ & 2.09 & 1.28 & 1.52 & 0.69 & 1.58 & 0.46 \\
\hline $16: 0$ & 14.86 & 6.26 & 14.43 & 2.69 & 14.36 & 169 \\
\hline $17: 0$ & 1.83 & 0.76 & 1.64 & 0.30 & 1.30 & 0.22 \\
\hline $18: 0$ & 3.12 & 1.36 & 3.54 & 1.46 & 2.95 & 0.63 \\
\hline $20: 0$ & 0.68 & 0.22 & 1.18 & 0.57 & 1.10 & 0.42 \\
\hline $22: 0$ & 0.36 & 0.11 & 0.42 & 0.27 & 0.49 & 0.18 \\
\hline $24: 0$ & 0.40 & 0.15 & 0.36 & 0.21 & 0.44 & 0.15 \\
\hline Sum SAFA & \multicolumn{2}{|c|}{24.95} & \multicolumn{2}{|c|}{24.58} & \multicolumn{2}{|c|}{24.58} \\
\hline i14:0 & 0.69 & 0.47 & 0.55 & 0.47 & 1.02 & 0.47 \\
\hline$i 15: 0$ & 5.02 & 1.14 & 4.04 & 1.56 & 6.40 & 1.54 \\
\hline a $15: 0$ & 8.07 & 1.76 & 6.23 & 2.62 & 10.86 & 2.47 \\
\hline i16:0 & 2.38 & 0.55 & 2.55 & 0.77 & 4.36 & 1.14 \\
\hline i17:0 & 1.25 & 0.36 & 1.38 & 0.13 & 1.61 & 0.23 \\
\hline $17: 1 \omega 8 \mathrm{c} / \mathrm{a} 17: 0$ & 4.53 & 1.51 & 3.98 & 0.65 & 4.59 & 0.59 \\
\hline Sum BRSAFA & \multicolumn{2}{|c|}{22.53} & \multicolumn{2}{|c|}{19.47} & \multicolumn{2}{|c|}{29.85} \\
\hline a $16: 0 / 16: 1 \omega 9 c$ & 0.65 & 0.28 & 0.66 & 0.28 & 0.70 & 0.07 \\
\hline $16: 1 \omega 7 c$ & 8.20 & 5.21 & 8.15 & 2.72 & 5.97 & 2.83 \\
\hline $16-107 t$ & 0.62 & 0.17 & 0.55 & $0.11 \frac{1}{x}$ & 0.50 & 0.14 \\
\hline $16: 1 \omega 5 c$ & 1.65 & 0.57 & 1.52 & 0.25 & 1.65 & 0.21 \\
\hline $16: 1 \omega 13 t$ & 0.16 & 0.35 & 0.20 & 0.11 & 0.10 & 0.10 \\
\hline $17: 1 \omega 6 c$ & 0.93 & 0.42 & 0.79 & 0.24 & 0.53 & 0.25 \\
\hline cy17:0 & 0.81 & 0.25 & 0.69 & 0.12 & 0.85 & 0.11 \\
\hline $18: 1 \omega 9 c$ & 3.39 & 1.35 & 3.30 & 0.53 & 2.11 & 0.65 \\
\hline $18: 1 \omega 7 \mathrm{c}$ & 10.26 & 4.98 & 10.17 & 1.79 & 7.97 & 1.89 \\
\hline cy19:0 $(\omega 7,8)$ & 0.70 & 0.21 & 0.97 & 0.31 & 0.87 & 0.18 \\
\hline Sum MUFA and $\mathrm{Cy}$ & \multicolumn{2}{|c|}{29.71} & \multicolumn{2}{|c|}{30.24} & \multicolumn{2}{|c|}{23.45} \\
\hline $16: 2 \omega 6 /$ br $15: 0$ & 0.46 & 0.29 & 0.47 & 0.11 & 0.56 & 0.10 \\
\hline poly 17 & 0.29 & 0.32 & 0.12 & 0.05 & 0.09 & 0.09 \\
\hline $18: 3 \omega 6$ & 0.68 & 0.23 & 0.93 & 0.16 & 0.78 & 0.14 \\
\hline $18: 4 \omega 3 / 12 \mathrm{me} 17: 0$ & 0.38 & 0.42 & 0.18 & 0.29 & 0.34 & 0.16 \\
\hline $18: 2 \omega 6$ & 0.39 & 0.28 & 0.75 & 0.25 & 0.34 & 0.22 \\
\hline $18: 3 \omega 3 /$ br $17: 1$ & 4.33 & 1.22 & 3.51 & 1.41 & 5.93 & 1.68 \\
\hline $20: 4 \omega 6$ & 2.74 & 2.82 & 2.90 & 1.33 & 1.16 & 0.90 \\
\hline $20: 5 \omega 3$ & 1.98 & 3.93 & 1.90 & 1.58 & 1.21 & 1.18 \\
\hline $20: 3 \omega 6$ & 0.27 & 0.18 & 0.64 & 1.00 & 0.12 & 0.08 \\
\hline $20: 403$ & 0.26 & 0.41 & 0.23 & 0.12 & 0.26 & 0.55 \\
\hline $20: 3 \omega 3$ & 0.41 & 0.44 & 0.99 & 0.75 & 0.52 & 0.19 \\
\hline $22: 5 \omega 6$ & 0.70 & 0.48 & 0.74 & 0.30 & 0.37 & 0.24 \\
\hline $22: 6 \omega 3$ & 0.74 & 1.32 & 1.23 & 1.57 & 0.53 & 0.69 \\
\hline $22: 4 \omega 6$ & 0.31 & 0.24 & 0.61 & 0.67 & 0.20 & 0.29 \\
\hline $22: 5 \omega 3$ & 0.18 & 0.29 & 0.29 & 0.37 & 0.16 & 0.25 \\
\hline poly 22 & 0.06 & 1.89 & 0.20 & 0.38 & 0.12 & 0.09 \\
\hline Sum PUFA & \multicolumn{2}{|c|}{15.16} & \multicolumn{2}{|c|}{16.67} & \multicolumn{2}{|c|}{13.02} \\
\hline 10me16:0 & 4.33 & 1.25 & 5.13 & 0.87 & 5.11 & 0.79 \\
\hline 10.11me18:0 & 1.09 & 0.37 & 1.53 & 0.67 & 1.48 & 0.38 \\
\hline Sum meBRSAFA & & & & & & \\
\hline Sum of PLFA listed & & & & & & \\
\hline Total pmol g ${ }^{-1}$ dry wt & 3411 & 2091 & 6524 & 5203 & 6489 & 4951 \\
\hline Cy/MUFA & & & & & & \\
\hline cy $17: 0 / 16: 1 \omega 7 \mathrm{c}$ & 0.11 & 0.04 & 0.09 & 0.04 & 0.16 & 0.07 \\
\hline cy $19: 0 / 18: 1 \omega 7 \mathrm{C}$ & 0.07 & 0.010 & 0.09 & 0.02 & 0.11 & 0.03 \\
\hline Trans/cis & & & & & & \\
\hline $16: 1 \omega^{7} \mathrm{t} / 16: 1 \omega^{7} \mathrm{C}$ & 0.08 & 0.010 & 0.09 & 0.03 & 0.12 & 0.03 \\
\hline $18: 1 \omega 7 \mathrm{t} / 18: 1 \omega 7 \mathrm{C}$ & 0.05 & 0.007 & 0.06 & 0.02 & 0.05 & 0.010 \\
\hline
\end{tabular}




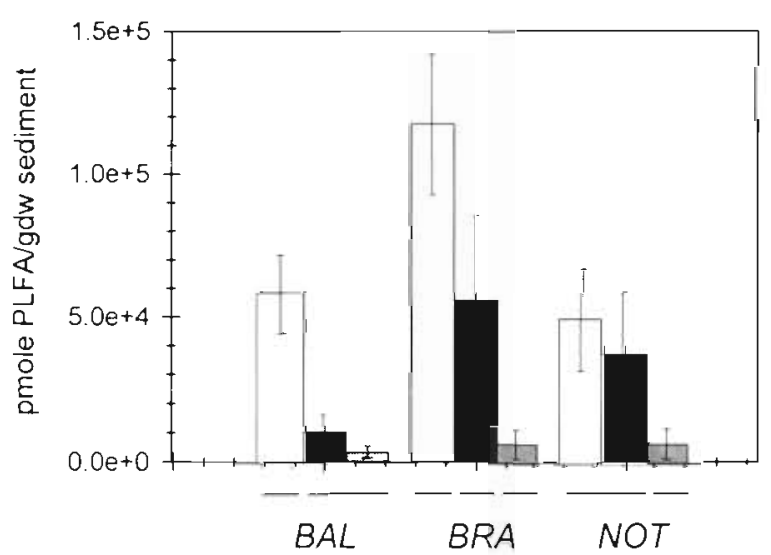

Fig. 1 Microbial biomass, as measured by total membrane PLFA for surface $(\square)$, worm burrow lining (ם), and subsurface (Wigiment sedimentes for 3 worm species. BAL: Balanoglossus aurantiacus; NOT Notomastus lobatus; BRA: Branchyoasychus americana. Values represent mean $\pm \mathrm{SD}$ for 10 samples. Lines under the $x$-axis are results of the Tukey test at a significance level of $\alpha=0.01$. Sample types connected by a common line are not significantly different. ANOVA-F significance was $\mathrm{p} \leq 0.0001$ for all groups, error $\mathrm{df}=27$

(Figs. $2 \& 3$ ). Only normal saturated PLFA did not differ significantly among the burrow lining samples. The significant difference between terminally branched saturated PLFA levels in Notomastus lobatus and Branchyoasychus americana burrows was largely due to

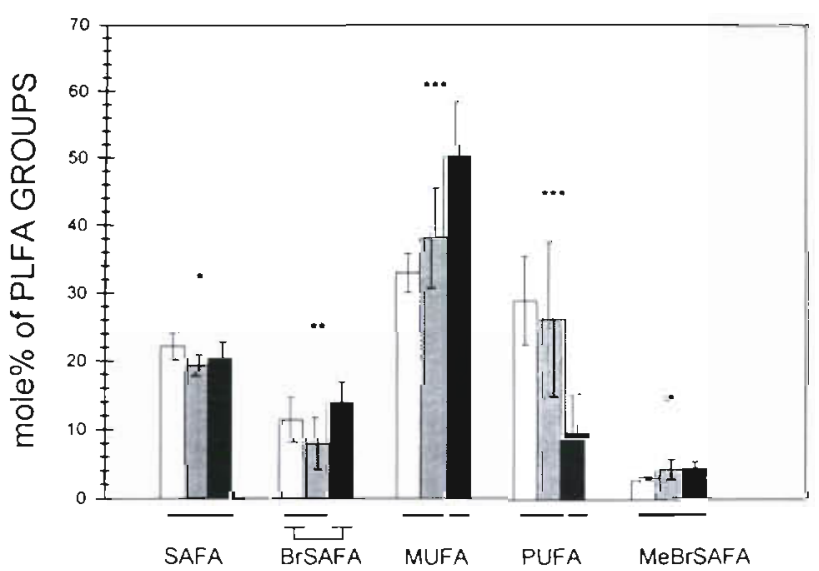

Fig. 2. Microbial community structure as represented by PLFA groups for burrow sediment sample sets collected from three different species of marine worms. Notomastus lobatus

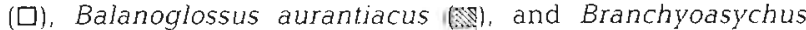
americana (ם). SAFA: saturated phospholipid fatty acids; BrSAFA: branched saturated PLFA; MUFA: monounsaturated PLFA; PUFA: polyunsaturated PLFA; MeBrSAFA: methyl branched saturated PLFA. See Fig. 1 legend for Tukey test description. ANOVA-F significance as follows: no star: no significant difference among groups, $p \leq 0.01, \cdot p \leq 0.001$, $\cdots p \leq 0.0001$. Error $\mathrm{df}=27$
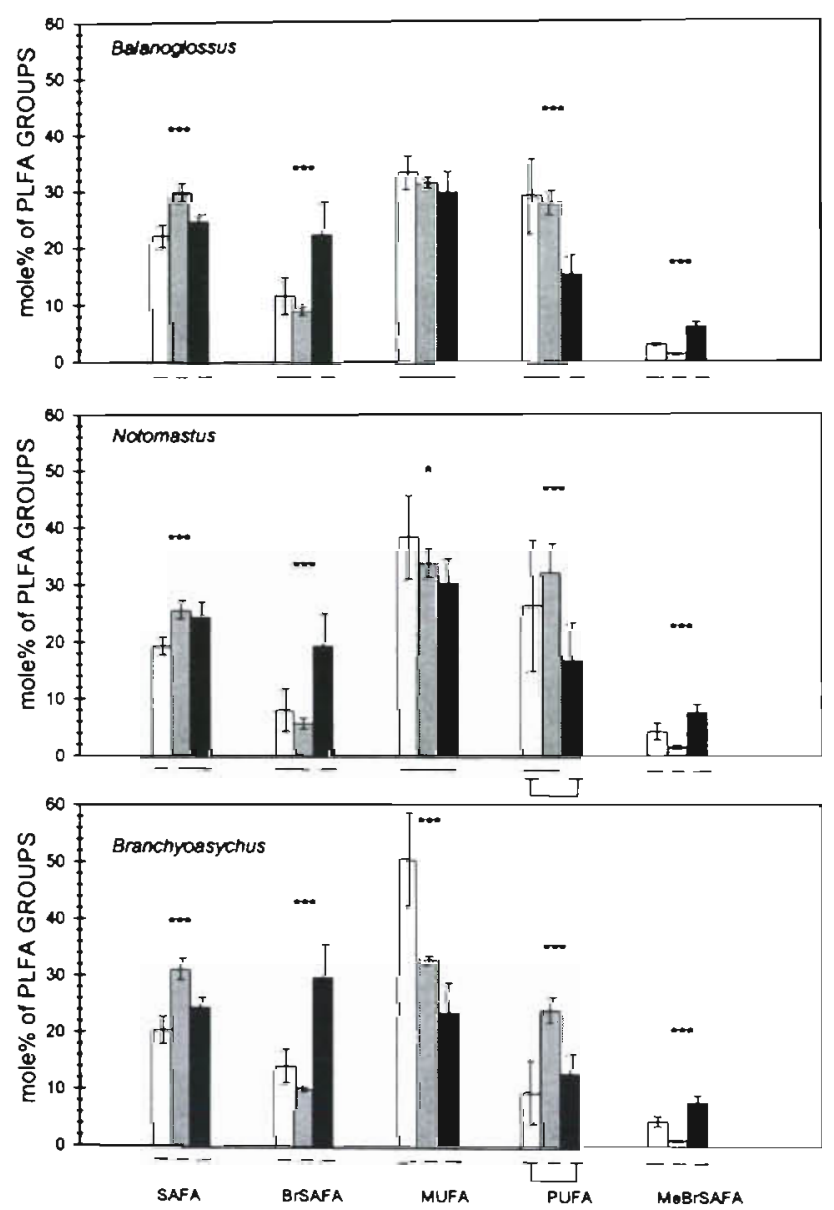

Fig. 3. Microbial community structure as represented by PLFA groups for sediment sample sets; burrow ( $\square$ ), surface (\$), and subsurface (ロ) sediments collected around Balanoglossus aurantiacus, Notomastus lobatus, and Branchyoasychus americana. See Fig. 1 \& 2 legends for Tukey test description, identification of PLFA groups, and ANOVA-F significance. Error $\mathrm{d} f=27$

higher amounts of i,a15:0 occurring in the $B$ americana burrows. Monounsaturated and cyclopropyl PLFA in $B$. americana burrows were significantly different from both Balanoglossus aurantiacus and N. lobatus burrow sediments. This was due to increased mole percentages of $16: 1 \omega 7 \mathrm{c}, 18: 1 \omega 7 \mathrm{c}$, and $c y 19: 0(\omega), 8)$ in the $B$. americana burrow sediments. PUFA were most abundant in (and not significantly different between) the B. aurantiacus and $N$. lobatus burrow sediments. PUFA levels in $B$ americana burrow sediments were significantly lower than in either of the other burrow types, due

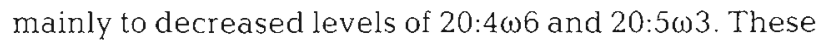
PUFA are abundant in eucaryotic organisms, with diatoms being the primary contributors in intertidal marine sediments (Erwin 1973, Orcutt \& Patterson 1975, Volkman et al. 1980, White 1983, Nichols et al. 1986, Smith et al. 1986). Methyl branched saturated 
fatty acids were significantly different among burrow sediments from the 3 species by ANOVA $(p \leq 0.01)$, but the Tukey test showed no significant differences among the 3 sample groups for these fatty acids.

\section{Multivariate cluster analysis}

Due to the size and complexity of the sample set, multivariate cluster analysis was used to relate samples or species based on the total PLFA profiles. Principal components analysis (PCA) was used to identify the variables (individual PLFA) which accounted for the majority of the variance among the PLFA profiles (Table 5). As was seen from the biomass and PLFA structural group evaluations, clusters constructed using PLFA mole percentage values for all 9 of the sample categories ( 3 worms $\times 3$ sediment types) showed that PLFA from the 3 types of sediments (surface, burrow, subsurface) were distinct (Fig, 4). The fidelity or accuracy of the clusters of sample types for the complete sample set was very high. The burrow ciusien inciuded 22 of the 30 burrow samples (similarity 0.51 ), and 1 subsurface sediment sample. This subsurface sample was characterized by elevated levels of $16: 1 \omega 7 \mathrm{c}, 20: 5 \omega 3$ and $20: 3 \omega 3$ relative to other subsurface samples in the set and may have been affected by the strong sediment mixing processes at this site (Grant 1983). Two other burrow samples, Branchyoasychus americana $\mathrm{B} 6$ and $\mathrm{B} 7$, clustered by themselves and contained very high mole percentages of $18: 1 \omega 7 \mathrm{C}$. This could be due to increased levels of either bacterial or microeucaryotic community members. The surface sediment cluster included 29 of the 30 surface sediment samples (similarity 0.67 ) and no members from other groups. The subsurface sediment cluster included 29 of 30 subsurface sediment samples (similarity 0.51). Two burrow samples, 1 Notomastus lobatus and 1 Balanoglossus aurantiacus, were also included in this subsurface cluster. These 2 burrow samples contained higher mole percentages of a 15:0 and lower mole percentages of $18: 1 \omega 7 \mathrm{c}, 20: 4 \omega 6,20: 5 \omega 3$ and $22: 6 \omega 3$, consistent with values seen in the other subsurface samples. It is likely that these samples included some subsurface sediment or were taken from vacant burrows with little or no burrow water circulation. In addition most sample sets collected for each worm species (i.e. burrow, surface and subsurface sediments for a species) also segregated into the same distinct clusters, though the fidelity of these clusters was lower, with more outliers and mixed clusters (Figs 5 to 7 ). This was likely due to the decreased statistical power resulting from separate analysis of the much smaller data sets from each species relative to the complete catalog of PLFA profiles from all species and sediment types. Most clusters contained 5 to 10 members, with some outliers. For example, N. lobatus samples B2 and B9 were outliers from the primary cluster of burrow samples from this set (Fig. 5). Similarly 3 B. aurantiacus subsurface sediment samples (D1, D3 and D4) were outliers from the primary cluster (Fig. 6).

\section{Principal components analysis}

PCA was used to identify a variety of PLFA which account for a significant portion of the variance found among the profiles (Table 5). PCA loadings for PLFA. incicate that monounsaturated fatty acids (MUFA), PUFA, branched saturated fatty acids, and 10me16:0 all represent important components of the sediment microbial communities. PCA loadings in conjunction with analysis of the mean PIFA values in the different sample sets were used to identify which PLFA explained the greatest level of variability among the different types of sediments. These PLFA included a 15:0, i15:0, 10me16:0, br $17: 1$, and $17: 1 \omega 8 \mathrm{c}$ for subsurface sediments, 20:503 and 16:107 $\mathrm{C}$ for burrow and

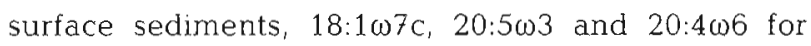
burrow sediments, and 16:0 and 14:0 for surface sediments. Many of these fatty acids were highly abundant in the samples (Tables 2 to 4). This information also forms the basis for analysis of indicator PLFA in the different sediment types.
Table 5. PLFA and their coefficients of loading from principal components analysis for principal component 1 (PC 1), principal component 2 (PC 2), and principal component 3 (PC 3) which together accounted for $89 \%$ of the variability within the data set. These were the 10 most variable components after analysis of all burrow, surface and subsurface sediment sample data from the Balanoglossus aurantiacus, Notomastus lobatus, and Branchyoasychus americana sample sets

\begin{tabular}{|c|c|c|c|c|c|}
\hline \multicolumn{2}{|c|}{$\begin{array}{c}\text { PC } 1 \\
50 \% \text { of variability }\end{array}$} & \multicolumn{2}{|c|}{$\begin{array}{c}\text { PC } 2 \\
34 \% \text { of variability }\end{array}$} & \multicolumn{2}{|c|}{$\begin{array}{c}\text { PC } 3 \\
5 \% \text { of variability }\end{array}$} \\
\hline $20: 5 \omega 3$ & 0.6066 & $18: 1 \omega 7 \mathrm{C}$ & 0.8342 & $16: 1 \omega^{7} \mathrm{c}$ & 0.5941 \\
\hline a 15:0 & -0.4053 & $16: 1 \omega)^{7} \mathrm{C}$ & 0.3016 & $16: 0$ & 0.3487 \\
\hline $16: 1 \omega 7 \mathrm{C}$ & 0.3993 & $20: 5 w 3$ & -0.2348 & $20: 4 \omega 6$ & -0.3109 \\
\hline $22: 6 \omega 3$ & 0.2379 & $c y 19.0$ & 0.1936 & $14: 0$ & 0.3021 \\
\hline 10 me16:0 & -0.2248 & a15:0 & -0.1665 & $20: 3 \omega 3$ & -0.2290 \\
\hline i15:0 & -0.2242 & $16: 0$ & -0.1599 & $18: 0$ & -0.2150 \\
\hline $18: 3 \omega 3$ & -0.2234 & $14: 0$ & -0.1260 & $20: 5 \omega 3$ & -0.2033 \\
\hline i16:0 & -0.1532 & $18: 3 \omega 3$ & -0.1062 & a 15:0 & 0.1973 \\
\hline $20: 4 \omega 6$ & -0.1280 & $15: 0$ & -0.0810 & $22: 6(t) 3$ & -0.1769 \\
\hline $16: 0$ & -0.1192 & $22: 6 \omega 3$ & -0.0807 & 115.0 & 0.1627 \\
\hline
\end{tabular}


Fig. 4. Dendrogram from multivariate cluster analysis, farthest neighbor complete linkage, of PLFA profiles from 90 samples collected around Notomastus lobatus (No), Balanoglossus aurantiacus (Ba), and Branchyoasychus americana $(\mathrm{Br})$ burrows, including 10 burrow sediment samples (B), 10 subsurface sediment samples (D), and 10 surface sediment samples (S) for each worm species. Line distances between clusters and indices indicate degree of similarity between clusters. An index of 1.000 indicates identical samples

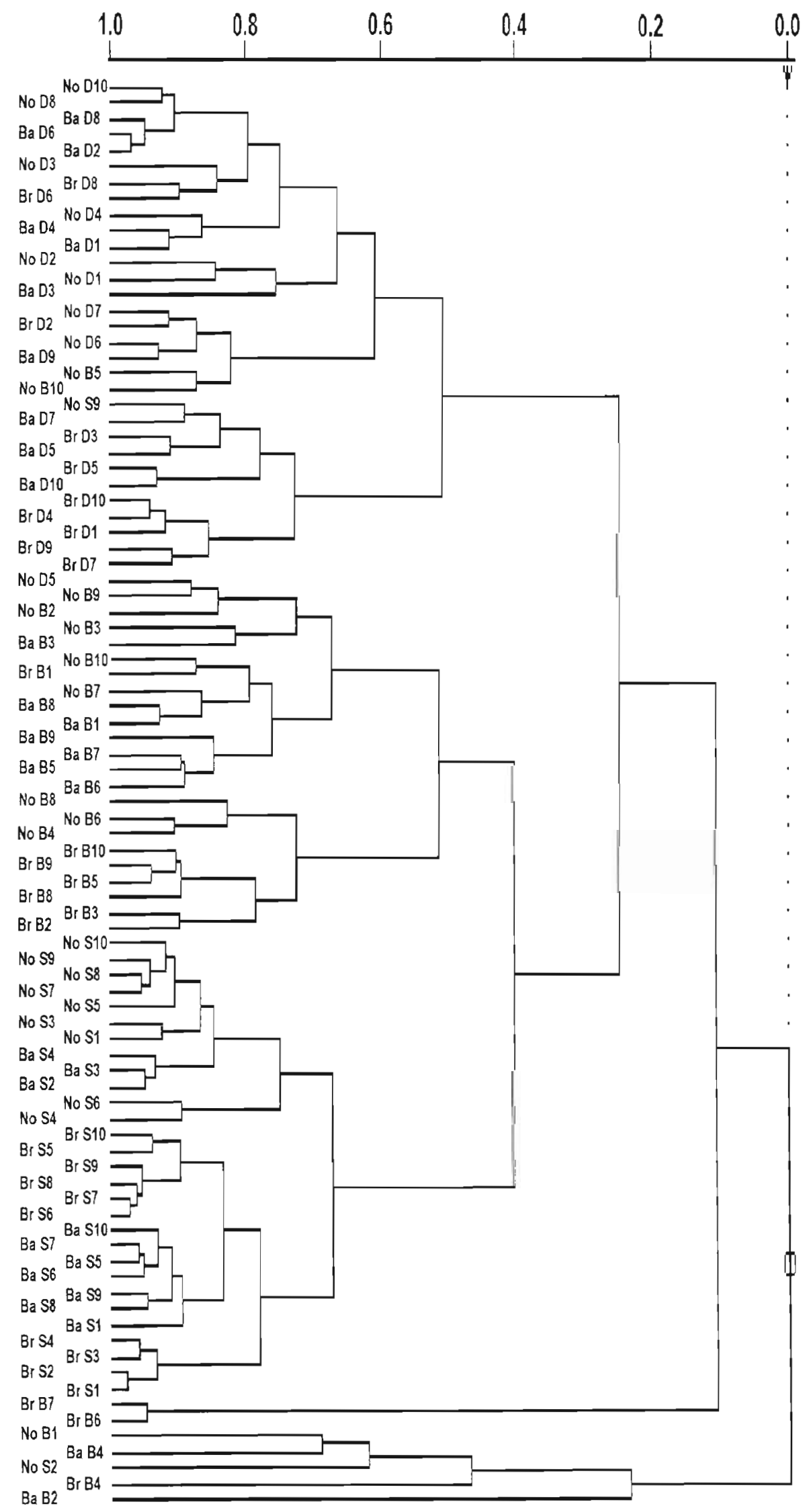




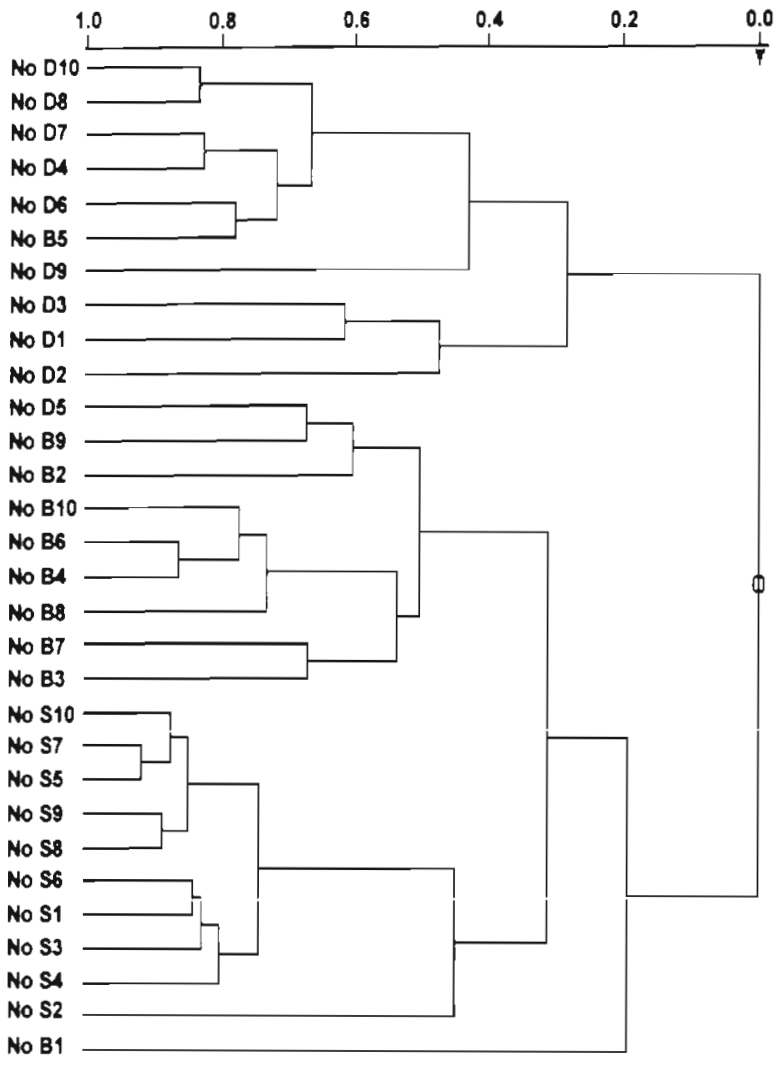

Fig. 5. Dendrogram from multivariate cluster analysis, farthest neighbor complete linkage, of PLFA profiles from a 30 sample set collected around Notomastus lobatus burrows. See Fig. 4 legend for details

\section{Biomarker PLFA}

Signature biomarker PLFA, which can be highly correlated with certain groups of microorganisms, were identified in all samples (Fig. 8). Several fatty acids have previously been used as indicators of bacterial biomass in marine sediments. Two particularly abundant PLFA in bacteria are 18:1 $17 \mathrm{c}$ and 16:1 $107 \mathrm{C}$ (Perry et al. 1979, Gillan et al. 1983, Parkes \& Taylor 1983, Guckert et al. 1985, Currie \& Johns 1988), although both of these MUFA are also found in eucaryotes (Johns et al. 1979, Gillan et al. 1981, Nichols et al. 1986). Total MUFA and cyclopropyl fatty acids in burrow samples ranged from 33 to $50 \mathrm{~mol} \%$ of the total and PUFA ranged from 9 to $29 \mathrm{~mol} \%$, with the highest ratio of MUFA:PUFA found in the Branchioasychus americana burrow sediments. The MUFA 18:107c and $16: 1 \omega 7 \mathrm{C}$ were abundant within the worm burrows, together accounting for 23 to $37 \%$ of the total PLFA abundance. They were also abundant in the surface (mean $=23 \%$ of total PLFA) and subsurface sediments $($ mean $=17 \%$ of total. PLFA $)$. Although we cannot dis- count that these 2 PLFA are found in microeucaryotic cell membranes, they are also terminal points in biosynthetic pathways utilized in all Gram-negative bacteria. It is expected that the presence of these PLFA is also due in large part to bacterial PLFA synthesis, providing evidence that bacteria contribute a significant component of microbial biomass in all 3 sediment types (Fig 8D)

PLFA indicative of sulfate reducers were identified in each of the sediment types examined (Fig. 8A). The abundance of 10me16:0, a major membrane component of the sulfate reducer Desulfobacter sp. (Kohring et al. 1994), as well as some other obligately anaerobic bacteria (Taylor \& Parkes 1983, Edlund et al. 1985, Dowling et al. 1986, 1988, Vainshtein et al. 1992), showed the same trends in all of the sample sets. Mole percentages increased in the order: surface sediments < burrow sediments < subsurface sediments (Fig. 8A). This branched saturated fatty acid accounted for 2.2 to $3.5 \%$ of the total PLFA in the burrow sediments. Other branched saturated PLFA are also indicative of anaerobic and sulfate reducing bacteria in marine sediments. Trends in the mole percentages for this group of

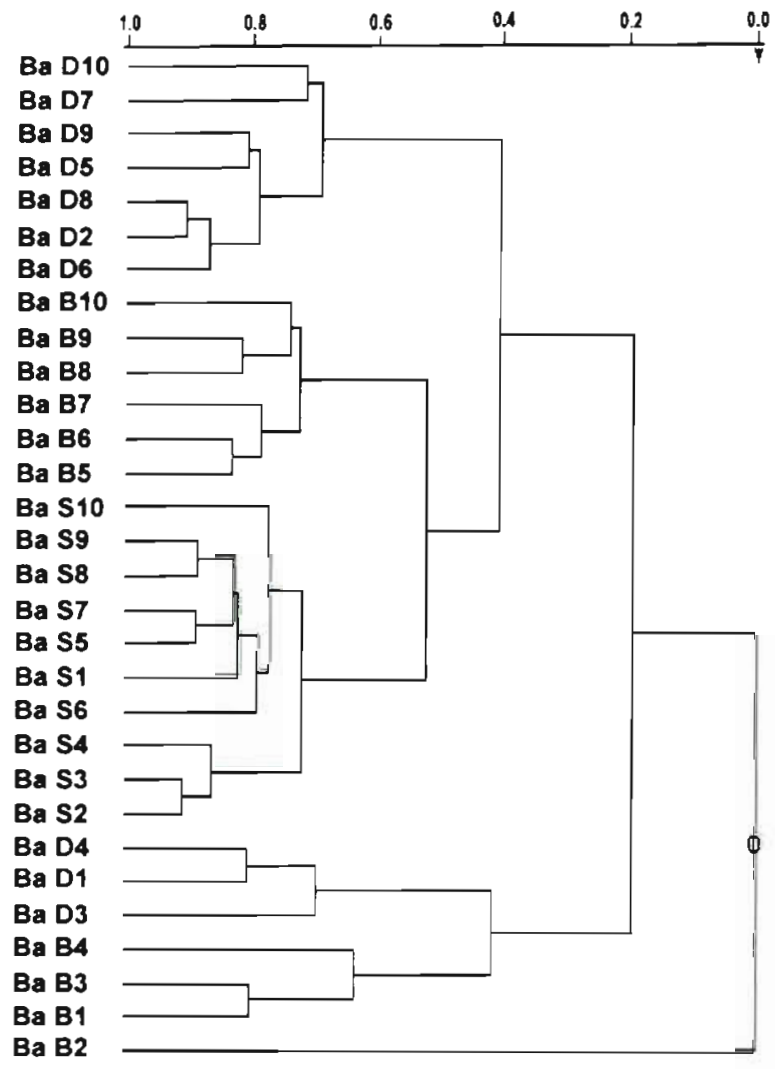

Fig. 6. Dendrogram from multivariate cluster analysis, farthest neighbor complete linkage, of PLFA profiles from a 30 sample set collected around Balanoglossus aurantiacus burrows. See Fig. 4 legend for details 


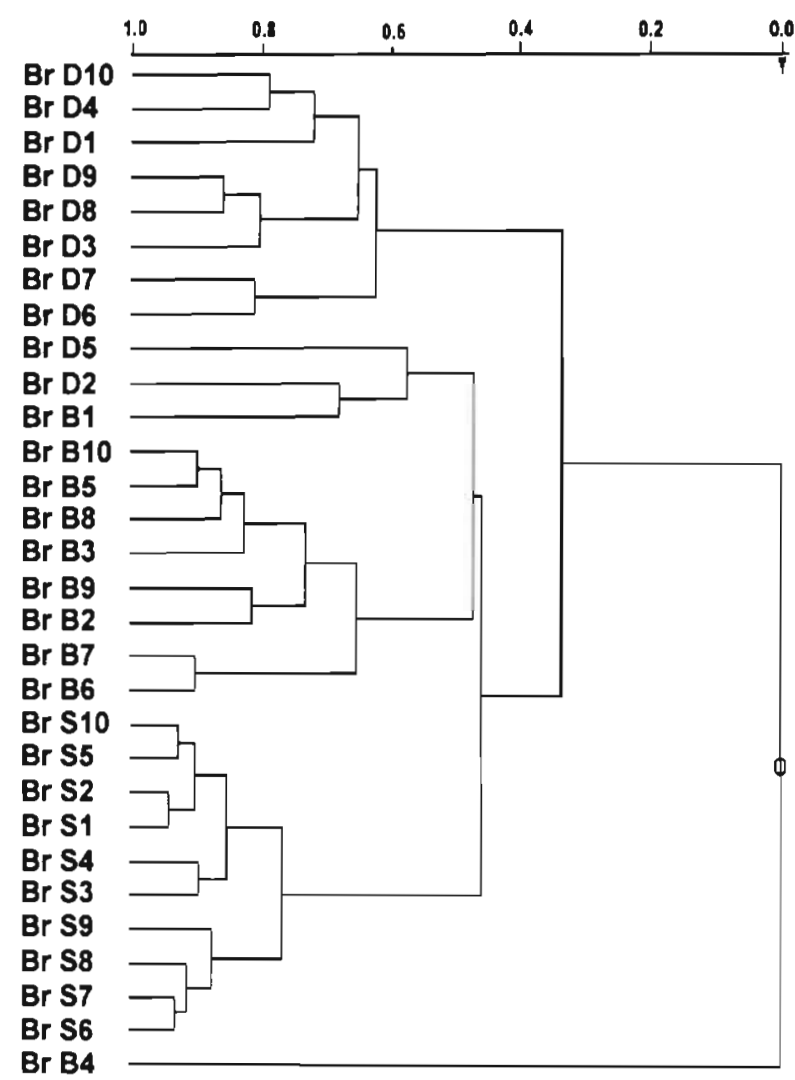

Fig. 7. Dendrogram from multivariate cluster analysis, farthest neighbor complete linkage, of PLFA profiles from a 30 sample set collected around Branchyoasychus americana burrow. See Fig. 4 legend for details
PLFA are the same as that of 10me16:0 (Fig. 8C). The high levels of PLFA indicative of sulfate reducers found in the subsurface sediments are consistent with sulfate reducer ecology and with other studies of marine sediments (Perry et al. 1979, Jørgensen 1980, Parkes \& Taylor 1983, Findlay et al. 1985, 1990, Dobbs \& Guckert 1988a, b). The presence of sulfate reducing bacteria in surface sediments can be explained by the presence of reduced microniches, such as fecal pellets (Jørgensen 1977), in oxidized marine sediments. Worm burrow lining sediments are organically enriched, irrigated with overlying seawater containing substantial levels of sulfate, and contain anaerobic microzones, making them an excellent niche for sulfate reducing bacteria (Aller 1978). Interestingly, the tightly consolidated and apparently poorly irrigated tubes of Branchioasychus americana contained high levels of branched saturated PLFA, indicative of sulfate reducers and other anaerobic bacteria.

PUFA with chain lengths of C19 and longer are considered biomarkers for eucaryotic organisms, particularly diatoms in estuarine sediments (Erwin 1973, Orcutt \& Patterson 1975, Volkman \& Johns 1977, Volkman et al. 1980, Gillan et al. 1981, Nichols et al. 1986, Smith et al. 1986, Vestal \& White 1989). Eucaryotic biomass, indicated by the abundance of the PLFA $16: 2 \omega 6,20: 4 \omega 6,20: 5 \omega 3,20: 3 \omega 3,22: 6 \omega 3$ and $22: 5 \omega 3$, showed the same trends in all sample sets, decreasing in the order surface $\geq$ burrow $\geq$ subsurface (Fig. 8B). This distribution of PUFA is consistent with
Fig. 8. Distribution of specific microbial groups around burrows of 3 species of infaunal worms. Notomastus lobatus () Balanoglossus aurantiacus (刃), Branchyoasychus americana (ם). (A) Sulfate reducing and other anaerobic bacteria based on 10me16:0. a signature lipid biomarker for Desulfobacter sp. (B) Eucaryotic organisms (primarily diatoms) based on 16:2w6, $20: 4 \omega 6,20: 5 \omega 3,20: 3 \omega 3,22$ $6 \omega 3$ and 22:5w3. (C) Grampositive bacteria based on i15:0, a15:0, i16:0, and i17:0. (D) Gram-negative bacteria based on monounsaturated PLFA (excluding 18:1 $1 \mathrm{9c}$, the eucaryotic precursor to polyunsaturated PLFA) and cyclopropyl PLFA
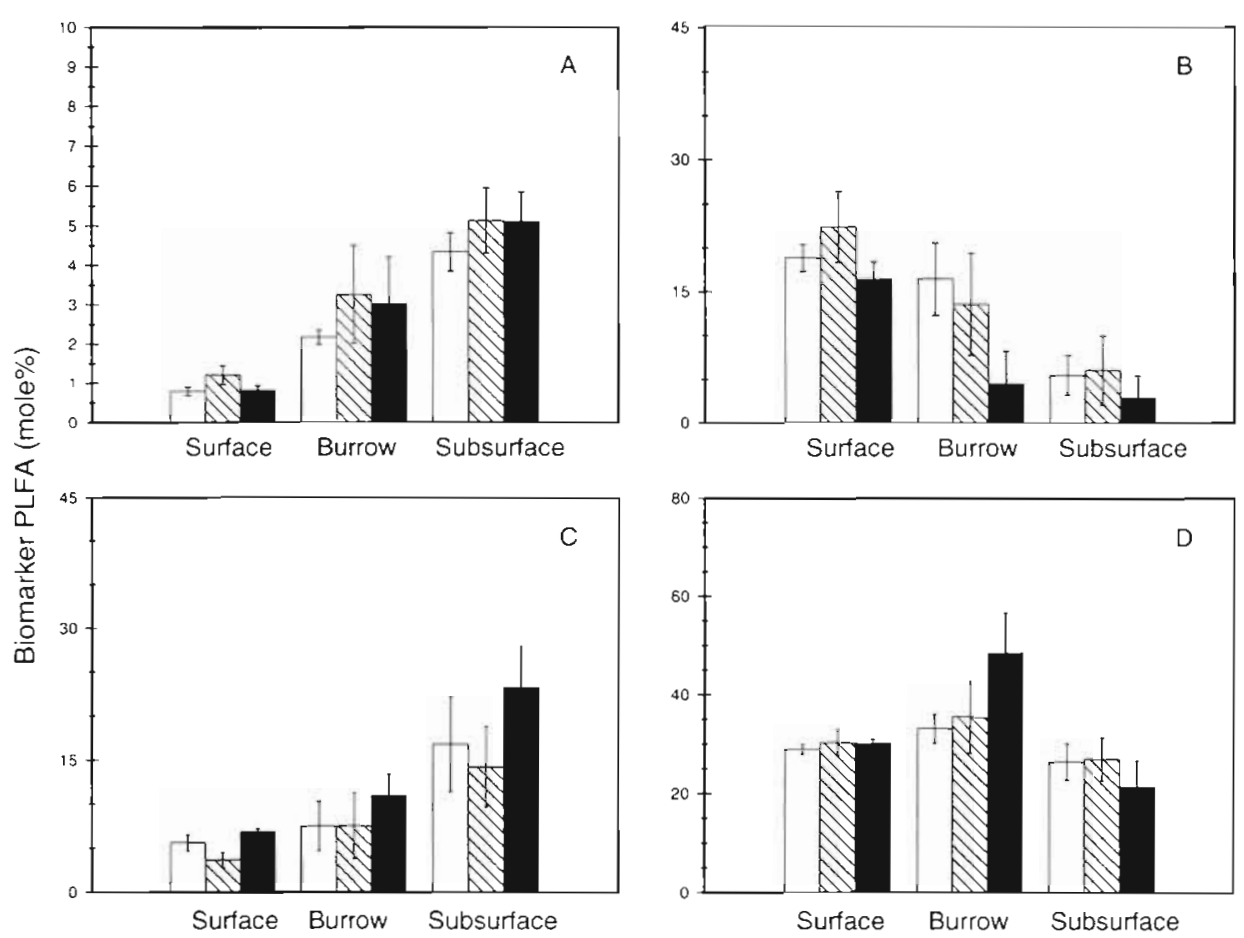
the known ecology of diatoms. Previous studies have found extensive and highly active microalgal communities at these and similar sites (Steward et al. 1992, Pinckney \& Zingmark 1993). The presence of low levels of diatom-indicator PLFA in subsurface sediments is consistent with reports of viable diatoms in anoxic, aphotic subsurface marine sediments (Wasmund 1989) and may represent diatoms buried by sediment mixing processes. As was the case for bacterial biomass, substantial viable eucaryotic biomass, as represented by PUFA, was found within the worm burrows. Diatoms and/or other eucaryotes could either be transported into the burrows by the feeding and irrigation activities of the worms, or simply fall or be washed into the burrow opening from surface sediments. The lower level of diatom PLFA biomarkers in the burrows of $B$. americana (Fig. $8 \mathrm{~B}$ ) could be explained by the elevated tube opening, which represents a physical barrier that could decrease surface sediment (and diatom) transport into the worm burrow.

\section{Microbial physiological status}

Previous investigators have used the ratios of trans to cis isomers of specific MUFA as indicators of bacteria growing under physiologically stressful conditions (Guckert et al, 1986, Findlay et al. 1990). The highest ratios of trans to cis fatty acids were found in subsurface sediments, although mean values there were only slightly higher than 0.1 , the lower limit for ratios typically interpreted to indicate stress (Tables 2 to 4). All burrow trans/cis ratios were lower than 0.1 , similar to those in the surface sediments. The higher ratios of trans to cis fatty acids seen in the subsurface sediments (Table 3) are consistent with data from other investigators (Guckert et al. 1986, Findlay et al. 1990) and are thought to reflect a common physiological adaptation to growth under oligotrophic conditions. High (>0.1) trans to cis ratios for the fatty acids

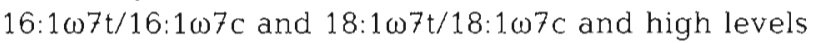
of cy 17:0 and cy 19:0 were also seen in anaerobic cultures enriched from marine sediment (Guckert et al. 1985). Much lower ratios were observed in the burrows, possibly reflecting the higher degree of organic enrichment there (Table 1). The presence of cyclopropyl fatty acids and the ratio of cyclopropyl to precursor MUFA (e.g cy 17:0/16:1w7c) have also been identified as indicators for physiological changes related to stress or anoxia (Guckert et al. 1985). The distribution of cyclopropyl fatty acids in our sediment samples is consistent with anoxia driven physiological changes and also refutes physiologically stressful conditions within the burrows. Cyclopropyl fatty acids and their associated ratios were typically highest in subsurface sediment samples, intermediate in burrow sediments and lowest in surface sediments (Tables 2 to 4). Branchyoasychus americana burrow sediments differed, having a high ratio of cy19:0/ $18: 1 \omega 7 \mathrm{c}$. This may be explained by the apparent stagnation of $B$. americana burrow water and the resulting anoxia of the tube sediments. The physiological status of the worm burrow microbial communities indicates an environment congenial to growth of bacteria, even when bromoaromatic exudates are present. Based on high levels of microbial biomass, substantial diversity (as reflected by the plethora of fatty acids identified in these samples), low stress indices, and the consistency of our results from burrows constructed by 3 different worms, the burrow environment appears to be highly conducive to bacterial growth.

\section{Concluding remarks}

Although the microbial communities in burrows of each of the worm species we examined have certain unique characteristics, these communities are still more similar to each other than to those in nearby surface and subsurface sediments. Undoubtedly the burrow microenvironment established by any infaunal species has properties resulting in greater or lesser representation of some bacterial groups. Which burrow features or host characteristics are most important in establishing the composition of the burrow lining microbial community are presently unknown. Production of bromometabolites by worms has been hypothesized to inhibit growth of aerobic bacteria in the burrows (Ashworth \& Cormier 1967, Sheikh \& Djerassi 1975, King 1986, Goerke \& Weber 1991), obviously altering the structure of the burrow microbial community. King (1988) reported such inhibition of aerobic bacteria in burrow sediments of the dibromophenol producer Saccoglossus kowalewskyi. This conclusion was based on low rates of glucose oxidation in contaminated sediments and absence of dibromophenol degradation by mixed aerobic cultures enriched from dibromophenol-contaminated sediments. However, our data, which reflect in situ biomass and community structure of undisturbed burrow lining biofilms, show no significant impact of biogenic bromophenols on microbial biomass, activities, or community structure. Surface and subsurface wormbed sediment communities were similarly unaffected (Steward et al. 1992). Determining which burrow and macrofaunal host features control the formation and composition of the burrow lining biofilm community will require further investigation. 
Acknowledgements. We acknowledge Sarah Woodin for identifying worms, Sarah Woodin and David Lincoln for helping identify and quantify bromometabolites and for their comments on this manuscript, and Michael Walla for GC/MS identification of bromoaromatic compounds. Helpful discussions of burrow properties with Roberta Marinelli are also acknowledged, as are the comments of anonymous reviewers. This research was supported by National Science Foundation grants BSR-8906705 and OCE-9201857 to C.R.L., and grants in aid to C.C.S. from the Slocum-Lunz Foundation. A preliminary account of this research was presented at the 93rd General Meeting of The American Society for Microbiology, Atlanta, Georgia, USA

\section{LITERATURE CITED}

Aller RC (1978) Experimental studies of changes produced by deposit feeders on pore water, sediment, and overlying water chemistry. Am J Sci 278:1185-1234

Aller RC (1980) Quantifying solute distributions in the b1oturbated zone of marine sediments by defining an average microenvironment. Geochim cosmochim Acta 44: $1955-1965$

Aller RC (1983) The importance of the diffusive permeability of animal burrow linings in determining marine sediment chemistry. J mar Res 41:299-322

Aller RC, Yingst JY (1978) Biogeochemistry of tube dwellings a study of the sedentary polychaete Amphitrite ornata $\mathrm{J}$ mar Res 36:201-254

Alongi DM (1985) Microbes, meiofauna, and bacterial productivity in tubes constructed by the polychaete Capitella capitata. Mar Ecol Prog Ser 23:207-208

Ashworth RB, Cormier MJ (1967) Isolation of 2,6-dibromophenol from the marine hemichordate, Balanoglossus biminiensis. Science 155:1558-1559

Baird BH, Nivens DE, Parker JH, White DC (1985) The biomass and community structure and spatial distribution of the sedimentary microbiota from a high-energy area of the deep sea. Deep Sea Res 32:1089-1099

Baird BH, White DC (1985) Biomass and community structure of the abyssal microbiota determined from the esterlinked phospholipids recovered from Venezuela Basin and Puerto Rico Trench sediment. Mar Geol 68:217-231

Balkwill DL, Leach FR, WIlson JT, MCNabb JF, White DC (1988) Equivalence of microbial biomass measures based on membrane lipid and cell wall components, adenosine triphosphate, and direct counts in subsurface aquifer sediments. Microb Ecol 16:73-84

Beukema JJ (1982) Annual variation in reproductive success and biomass of the major macrozoobenthic species living in a tidal flat area of the Wadden Sea. Neth J Sea Res 16: $37-45$

Beukema JJ, de Vlas J (1979) Population parameters of the lugworm, Arenicola marina, living on tidal flats in the Dutch Wadden Sea. Neth J Sea Res 13:331-353

Boudreau BP, Marinelli RL (1994) A modelling study of discontinuous biological irrigation. J mar Res 52:947-968

Chen YP, Lincoln DE, Woodin SA, Lovell CR (1991) Purification and properties of a unique flavin-containing chloroperoxidase from the capitellid polychaete Notomastus lobatus. J Biol Chem 266:23909-23915

Christie WW (1989) Gas chromatography and lipids. The Oily Press, Ayr

Corgiat JM, Dobbs FC, Burger MW, Scheuer PJ (1993) Organohalogen constituents of the acorn worm Ptychodera bahamensis. Comp Biochem Physiol 106B:83-86
Currie BR, Johns RB (1988) Lipids as indicators of the origin of organic matter in fine marine particulate matter. Aust $J$ mar Freshwat Res 39:371-383

Dame R, Chrzanowski T, Bildstein K, Kjerfve B, McKellar H, Nelson D, Spurrier J, Stancyk S, Stevenson H, Vernberg J, Zingmark R (1986) The outwelling hypothesis and North Inlet, South Carolina. Mar Ecol Prog Ser 33:217-229

Dobbs FC, Guckert JB (1988a) Callianasa trilobata (Crustacea: Thalassinidea) influences abundance of meiofauna and biomass, composition, and phs siologic state of microbial communities within its burrow. Mar Ecol Prog Ser 45 : $69-79$

Dobbs FC, Guckert JB (1988b) Microbial food resources of the macrofaunal-deposit feeder Ptychodera bahamensis (Hemichordata: Enteropneusta). Mar Ecol Prog Ser 45: $127-136$

Dowling NJE, Nichols PD, White DC (1988) Phospholipid fatty acid and infra-red spectroscopic analysis of a sulphate-reducing consortium. FEMS Microbiol Ecol 53: $325-334$

Dowling NJE, Widdel F, White DC (1986) Phospholipid esterlinked fatty acid biomarkers of acetate-oxidizing sulphatereducers and other sulphide-forming bacteria. J Gen Microbiol 132;1815-1825

Edlund A, Nichols PD, Roffey R, White DC (1985) Extractable and lipopolysaccharide fatty acid hydroxy acid profiles from Desulfovibrio species. J Lipid Res 26:982-988

Erwin JA (1973) Comparative biochemistry of fatty acids in eukaryotic microorganisms. In: Erwin JA. (ed) Lipids and biomembranes of eukaryotic microorganisms. Academic Press, New York, p 41-143

Federle TW, Dobbins DC, Thornton-Manning JR, Jones DD (1986) Microbial biomass, activity, and community structure in subsurface soils. Groundwater 24:365-374

Fenchel TM, Reidel RJ (1970) The sulfide system: a new biotic community underneath the oxidized layer of marine sand bottoms. Mar Biol 7:255-268

Fielman KT, Targett NM (1995) Variation of 2,3,4-tribromopyrrole and its sodium sulfamate salt in the hemichordate Saccoglossus kowalevskii. Mar Ecol Prog Ser 116:125-136

Findlay RH, Pollard PC, Moriarty DJW, White DC (1985) Quantitive determination of microbial activity and community nutritional status in estuarine sediments: evidence for a disturbance artifact. Can J Microbiol 31:493-498

Findlay RH, Trexler B, Guckert JB, White DC (1990) Laboratory study of disturbance in marine sediments: response of a microbial community. Mar Ecol Prog Ser 62:121-133

Gillan FT, Johns RB, Verhey TkV, Nichols PD (1983) Monounsaturated fatty acids as specific bacterial markers in marine sediments. In: Bjoroy $M$ (ed) Advances in organic chemistry. Wiley and Sons Ltd, New York, p 198-206

Gillan FT, McFadden Gl, Wetherbee R, Johns RB (1981) Sterols and fatty acids of an Antarctic sea ice diatom, Stauronels amphioxys. Phytochemistry 20:1935-1937

Goerke H. Weber K (1991) Bromophenols in Lanice conchilega (Polychaeta, Terebellidae): the influence of sex, weight and season. Bull mar Sci 48:517-523

Grant J (1983) The relative magnitude of biological and physical sediment reworking in an intertidal community. $J$ mar Res 41:673-689

Guckert JB, Antworth CP, Nichols PD. White DC (1985) Phospholipid, ester-linked fatty acid profiles as reproducible assays for changes in prokaryotic community structure of estuarine sediments. FEMS Microbiol Ecol 31:147-158

Guckert JB, Hood MA, White DC (1986) Phospholipid ester linked fatty acid profile changes during nutrient deprivation of Vibrio cholerae: increases in the trans/cis ratio and 
proportions of cyclopropyl fatty acids. Appl Environ Microbiol 52:794-801

Guckert JB, Ringelberg DB, White DC, Hanson RS, Bratina BJ (1991) Membrane fatty acids as phenotypic markers in the polyphasic taxonomy of methylotrophs within the proteobacteria. J Gen Microbiol 137:2631-2641

Haack SK, Garchow H, Odelson DA, Forney LJ, Klug M.J (1994) Accuracy, reproducibility and interpretation of fatty acid methyl ester profiles of model bacterial communities. Appl Environ Microbiol 60:2483-2493

Higa T, Scheuer PJ (1975a) 3-Chloroindole, principal odorous constituent of the hemichordate Ptychodera flava laysanica. Naturwissenschaften 62:395-396

Higa $T$, Scheuer PJ (1975b) Constituents of the marine annelid Thelepus setosus. Tetrahedron 31:2379-2381

Jensen P, Emrich R, Weber K (1992) Brominated metabolites and reduced numbers of meiofauna organisms in the burrow wall lining of the deep-sea enteropneust Stereobalanus canadensis. Deep Sea Res 39:1247-1253

Johns RB, Nichols PD, Perry GJ (1979) Fatty acid composition of ten marine algae from Australian waters. Phytochemistry $18: 799-802$

Jørgensen BB (1977) Bacterial sulfate reduction within reduced microniches of oxidized marine sediments. Mar Biol. 41:7-17

Jørgensen BB (1980) Mineralization and the bacterial cycling of carbon, nitrogen and sulphur in marine sediments. In: Ellwood DC, Hedger JH, Latham MJ, Lynch JM, Slater JH (eds) Contemporary microbial ecology. Academic Press, New York, p 239-251

Kates $M$ (1.986) Techniques in lipidology: isolation, analysis and identification of lipids, 2nd edn. Elsevier, Amsterdam

King GM (1986) Inhibition of microbial activity in marine sediments by a bromophenol from a hemichordate. Nature $323: 257-259$

King GM (1988) Dehalogenation in marine sediments containing natural sources of halophenols. Appl Environ Microbiol 54:3079-3085

Kohring LL, Ringelberg DB, Devereux R, Stahl DA, Mittelman MW, White DC (1994) Comparison of phylogenetic relationships based on phospholipid fatty acid profiles and ribosomal RNA sequence similarities among dissimilatory sulfate-reducing bacteria. FEMS Microbiol Lett 119: $303-308$

Krantzberg G (1.985) Influence of bioturbation in aquatic environments. Environ Pollut 39:99-122

Lappin-Scott HM, Costerton JW (1989) Bacterial biofilms and surface fouling. Biofouling 1:323-342

Mangum CP (1964a) Activity patterns in metabolism and ecology of polychaetes. Comp Biochem. Physiol 11:239-256

Mangum CP (1964b) Studies on spectation in maldanid polychaetes of the North American Atlantic Coast. II. Distribution and competitive interaction of five sympatric species. Limnol Oceanogr 9:12-26

Nichols PD, Palmisino AC, Smith GA, White DC (1986) Lipids of the antarctic sea ice diatom. Nitzschia cylindrus. Phytochemustry 25:1649-1653

Orcutt DM, Patterson GW (1975) Sterol, fatty acid and elemental composition of diatoms grown in chemically defined medic. Comp Biochem Physiol 50B:579-583

Parkes RJ, Taylor J (1983) The relationship between fatty acid distributions and bacterial respiration types in contemporary marine sediments. Estuar coast Shelf Sci 16:173-189

Perry GJ, Volkman JK, Johns RB (1979) Fatty acids of bacterial origin in comtemporary marine sediments. Geochim Cosmochim Acta 43:1715-1725

Pinckney J, Zingmark R (1993) Biomass and production of benthic microalgal communities in five typica] estuarine habitats. Estuaries 16:887-897

Powell EN (1977) The relationshıp of the trace fossil Gyrolithe (= Xenolith) to the family Capitellidae (Polychaeta). J Paleontol 51:552-556

Rajendran N, Suwa Y, Urushigawa Y (1992) Microbial community structure in sediments of a polluted bay as determined by phospholipid ester-linked fatty acids. Mar Pollut Bull 24:305-309

Ringelberg DB, Davis JD, Smith GA, Pfiffner SM, Nichols PD, Nickels JS, Henson JM, Wilson JT, Yates M, Kampbell DH, Read HW, Stocksdale TT, White DC (1986) Validation of signature polarlipıd fatty acid biomarkers for alkaneutilizing bacterial in soils and subsurface aquifer materials. FEMS Microbıl Ecol 62:39-50

Ruppert EE, Fox RS (1988) Seashore animals of the Southeast. University of South Carolina Press, Columbia

Schottler U, Weinhausen G, Zebe E (1983) The mode of energy production in the lugworm Arenicola marina at different oxygen concentrations. J Comp Physiol 149B:547-555

Sheikh YM, Djerassi C (1975) 2,6-dibromophenol and 2,4,6tribromophenol - antiseptic secondary metabolites of Phoronopsis viridis. Experientia 31:265-392

Smith GA, Nichols PD, White DC (1986) Fatty acid composition and microbial activity of benthic marine sediment from McMurdo Sound, Antarctica. FEMS Microbiol Ecol 38:219-231

Steward CC, Dixon TC, Chen YP, Lovell CR (1995) Enrichment and isolation of a reductively debrominating bacterium from the burrow of a bromometabolite producing marine hemichordate. Can J Microbiol 41:637-642

Steward CC, Pinckney J, Piceno Y, Lovell CR (1992) Bacterial numbers and activity, microalgal biomass and productivity and meiofaunal distribution in sediments naturally contaminated with biogenic bromophenols. Mar Ecol Prog Ser 90:61-72

Taylor J, Parkes RJ (1983) The cellular fatty acids of the sulphate-reducing bacteria, Desulfobacter sp., Desulfobulbus sp., and Desulfovibrio desulfuricans. J Gen Microbiol 1.29:3303-3309

Tunlid A, White DC (1992) Biochemical analysis of biomass, community structure, nutritional status, and metabolic activity of microbral communities in soil. In: Stotsky G, Bollag JM (eds) Soil biochemistry. Marcel Dekker Inc, New York, p 229-262

Vainshtein M, Hippe H, Kroppenstedt RM (1992) Cellular fatty acid composition of Desulfovibrio species and its use in classification of sulfate-reducing bacteria. Syst Appl Microbiol 15:554-566

Vestal RJ, White DC (1989) Lipid analysis in microbial ecology: quantitative approaches to the study of microbial communities. BioSci 39:535-541

Volkman JK, Johns RB (1977) The geochemical significance of positional isomers of unsaturated acids from an intertidal zone sediment. Nature 267:693-694

Volkman JK, Johns RB, Gillan FT, Perry GJ, Bavor HJ (1980) Microbial lipids of an intertidal sediment. I. Fatty acids and hydrocarbons. Geochim Cosmochim Acta 44: $1133-1143$

Wasmund N (1989) Micro-autoradiographic determination of the viability of algae inhabiting deep sediment layers. Estuar coast Shelf Sci 28:651-656

White DC (1983) Analysis of microorganisms in terms of quantity and activity in natural environments. In: Slater $\mathrm{JH}$, Whittenbury $\mathrm{R}_{1}$ Wimpenny JWT (eds) Microbes in their natural environments. Symp Soc Gen Microbiol 34 Cambridge University Press, Cambridge, p 37-66 
White DC (1988) Validation of quantitative analysis for microbial biomass, communty structure, and metabolic activity Arch Hydrobiol Beih Ergebn Limnol 31:1-18

White DC, Davis WM, Nichols JS, King JD, Bobbie RJ (1979) Determination of the sedimentary microbıal biomass by extractable lipid phosphate. Oecologia 40:51-62

This article was submitted to the editor
Woodin SA, Marinelli R, Lincoln DE (1993) Allelochemical inhibition of recruitment in a sedimentary assemblage. $J$ Chem Ecol 19:517-530

Woodin SA, Walla MD, Lincoln DE (1987) Occurrence of brominated compounds in soft-bottom benthic organisms. J exp mar Biol Ecol 107:209-217

Manuscript first received: August 8, 1994

Revised version accepted: August 23, 1995 